\title{
Multiscale Description and Upscaling of Fluid Flow in Subsurface Reservoirs
}

\author{
B. Nœetinger ${ }^{1}$ and G. Zargar ${ }^{1,2}$ \\ 1 Institut français du pétrole, 1 et 4 avenue de Bois-Préau, 92852 Rueil-Malmaison Cedex - France \\ 2 National Iranian South Oil Company Ahwaz, 61335 - Iran \\ e-mail: benoit.noetinger@ifp.fr - ghasem.zargar@ifp.fr
}

\begin{abstract}
Résumé - Description multiéchelle des écoulements dans les réservoirs souterrains - Les formations géologiques sont des objets naturels complexes, sièges d'innombrables processus géologiques, mécaniques, physico-chimiques se déroulant sur des échelles d'espace et de temps extrêmement larges. Ainsi, des propriétés de mouillabilité directement issues de phénomènes moléculaires influencent directement les déplacements eau-huile, et donc évidemment la récupération finale du pétrole. De même, les hétérogénéités du réservoir, couplées aux non-linéarités de la mécanique des fluides, jouent un rôle essentiel pour localiser les écoulements dans des chenaux préférentiels.

De façon à gérer cette complexité, et à hiérarchiser au mieux l'influence des nombreux phénomènes et paramètres, il est essentiel de disposer d'une description multiéchelle du transport de fluides dans ces milieux. Ceci permet aux ingénieurs en géosciences de travailler un modèle de terre partagé, réceptacle du savoir géologique, géophysique, ainsi que des données de gisement permettant l'intégration des divers métiers. On peut ainsi se concentrer sur les principaux phénomènes contrôlant l'écoulement de l'huile, et donc, sa récupération. Ceci aide les ingénieurs à intégrer les données issues de mesures de natures diverses à des échelles différentes et à diminuer les incertitudes, permettant ainsi la prise de meilleures décisions économiques.

Dans cet article, nous présentons la philosophie globale des techniques de changement d'échelle, en y incluant la description des concepts les plus récents d'approches multiéchelles.
\end{abstract}

\begin{abstract}
Multiscale Description and Upscaling of Fluid Flow in Subsurface Reservoirs - Natural geological formations are complex objects, involving geological, mechanical, physico-chemical processes occurring over very wide length scales and time scales. Phenomena ranging from the molecular scale to several hundred of kilometers may influence the overall behaviour of fluid transport in a geological formation. For example, wettability properties, themselves due to molecular effects, have a very strong impact on the water/oil displacements in oil reservoirs. Analogously, reservoir heterogeneities that cover a large range of spatial scales play an essential role to channel fluid-flows, especially when they are coupled with non linearities inherent to fluid dynamics.

In order to face this complexity, and to be able to hierarchize the influence of the various relevant geological and physico-chemical phenomena, it is thus essential to handle a multiscale description of fluid transport in these reservoirs. This is an essential tool to help reservoir engineers to focus on the crucial phenomena that control the flow. This helps them to integrate data, and this results in a lowering of the uncertainties of the reservoir description that enhances economical decisions.
\end{abstract}

In this paper, we present classical upscaling approaches, as well as more recent multiscale concepts. 


\section{NOMENCLATURE}

\section{Roman symbols}

$c_{t} \quad$ Total compressibility value $\left(\mathrm{bar}^{-1}\right)$

$c(\mathbf{r}, t) \quad$ Local tracer concentration $\left(\right.$ mole $\left.\mathrm{m}^{-3}\right)$

$C(\mathbf{r}) \quad$ Log permeability covariance function evaluated at lag $\mathbf{r}$

$C(\mathbf{q}) \quad$ Fourier transform of $C(\mathbf{r})$ at wave vector $\mathbf{q}$ $\left(\mathrm{m}^{\mathrm{D}}\right)$

$C_{\Gamma}(r) \quad$ Filtered log permeability covariance function

D Flow dimension $(\mathrm{D}=1,2$ or 3$)$

d(u) Dispersivity tensor (m)

$\mathbf{d}_{\text {obs }}$

$f(\mathbf{r}, t)$

$J(\mathbf{k})$

$k(\mathbf{r})$

$k_{v} / k_{h}$

$\mathbf{K}_{\text {eff }}$

$K_{g}$

$K_{g \Gamma}$

$k r_{i}(S)$

$l_{c}$

$l_{h} / l_{v}$

$l_{i j}$

$L$

$p(\mathbf{r})$

q

$r$

$R(t)$

$s_{i j}$

$S$

$\mathbf{u}(\mathbf{r})$

$t_{i j}$

$T_{I J}$

$Y(\mathbf{r})$ $\sigma_{\text {mod }}^{2}$

$\Sigma(\mathbf{r}, t)$

$\mu$

$\mu_{i}$

$\omega(\alpha)$
Numerical modelling errors variance

Diffusion kernel

Fluid viscosity

$i$ th fluid viscosity

Permeability averaging exponent.

\section{INTRODUCTION}

In this paper, we show the importance of having a multiple scale description of fluid flow in subsurface reservoirs. In the upstream oil industry (or when managing aquifers), the basic goal of reservoir simulation is to provide tools allowing engineers to perform accurate sensitivity studies, in order to optimise an oil recovery scenario, taking into account all the available data (Alabert, 1989; Deutsch and Journel, 1992; Blanc et al., 1996; Galli et al., 1990; Guérillot et al., 1991; Gorell and Basset, 2001). The data can be either of geological, geophysical nature, or coming from the reservoir exploitation itself such as a pressure variation, watercut or even repeated seismic acquisition indicating subsurface fluid displacements (Kretz et al., 2002; Pianelo et al., 2000). Ideally, the engineer wants to obtain production forecasts manipulating only the geological model, and changing the exploitation scenario, numerical aspects being managed by the simulator itself.

Even with all these data, the engineer wants to manage the remaining uncertainties inherent to any geoscience modelling. He is thus led to perform extensive sensitivity studies or even Monte-Carlo simulations of several (in practice up to 100 , in theory several thousands) reservoir realisations. To reach this goal, engineers must first transform the original 3D geological map into a numerical model well suited for solving the discrete equations arising from any numerical treatment of multiphase fluid flow equations. A basic issue is simply to be able to perform these calculations in an acceptable CPU time and accuracy. The simplest approach is thus to try to reduce the number of grid blocks and of unknowns to get a tractable problem. This is the classical issue of upscaling, or pseudoisation in the multiphase case, that is the subject of a huge amount of literature (see Ahmadi et al., 1993, 1996; Artus et al., 2003 and Renard and de Marsily, 1997 for a review). The basic question is thus to be able to take into account the small scale disorder of the medium (or "subgrid" effects in the language of people involved in Computational Fluid Dynamics, CFD see e.g. Lesieur and Métais, 1996) at this coarser scale. Depending on the academic background of the authors, the focus is on mathematical asymptotic theories of homogenisation and more recently on stochastic Partial Differential Equations (PDE) (Jikov et al., 1994). People having a more physical and fluid mechanic background will adopt methods arising from mechanics or physics such as volume averaging (Cherblanc, 1999; Quintard and Whitaker, 1994), effective 
medium (Dagan, 1989), percolation theory (Stauffer, 1985). Some perturbation methods, originally devised to treat questions arising from quantum mechanics, next adapted to study turbulent transport and including renormalisation group approaches, are also employed in the stochastic context (Avellaneda, 1996; Christakos et al., 1993, 1995; King, 1989; Notinger, 1994; Glimm et al., 1992; Zhang, 1992; Stepanayants and Teodorovitch, 2003). More recently, very interesting purely numerical techniques based on new finite elements approaches proposing a fully multiscale treatment were proposed (see e.g. Guérillot and Verdière, 1995; Gautier et al., 1999; Arbogast and Bryant, 2001; Hou and Wu, 1997, 1999). Analogous treatments can be found in the vast domain of CFD, where the Large Eddy Simulation (LES) technique of turbulent flows is very close in spirit to present considerations (Germano, 1996). We must also mention the considerable amount of applied reservoir engineering or hydrology literature proposing practical solutions that can be justified a posteriori using rigorous approaches. We defer the reader to specialised texbooks (Dagan, 1989 and references therein).

Another important topic is that these modern approaches of reservoir characterisation imply manipulating data that are intrinsically defined and measured over rather different support size, such as core plug permeability measurements of some $\mathrm{cm}$ or well tests interpretation at a $100 \mathrm{~m}$ scale. So one of the major concerns of reservoir engineers is integration of different data obtained by different measurement processes at different scales (Pianelo et al., 2000; Schaaf and Mezghani, 2002). A major issue is to be able to reconcile the geological model to various available dynamic data that give additional information about the considered reservoir. This generally implies solving inverse problems using optimisation procedures that need a great number of forward modelling evaluations. As we will see, to model flow in an oil reservoir is basically a formidable task: starting from physical laws that remain still under investigation at the pore scale (this is itself the first Darcy scale upscaling problem!), one must build a large scale model using data that are essentially measured at the wells. This corresponds to a negligible portion of the reservoir, so we are led to model a quasi unknown object with quasi unknown laws! Happily, this discouraging picture becomes more beautiful thanks to interesting features of many diffusive transport phenomena, called the self averaging property, whose direct consequence is that a complex system like an oil reservoir can sometimes be described at large scale by a rather small number of parameters (Goldenfeld, 1992; Koslov, 1993). Among those parameters, some are clearly related to small scale data, and others appear as the result of a complex combination of coupled effects of small scale physics with large scale heterogeneities. A typical example is the coupling of viscous instabilities of Saffman-Taylor type with preferential permeability streaks (Saffman and Taylor, 1958). This coupling can give rise to non trivial large scale behaviour that may be described by equations that are qualitatively different than the small scale Darcy equations (Langlo and Espedal, 1994; Lenormand and Thiele, 1994; Artus et al., 2003). One of the more important and difficult task is to predict and identify the occurrence of such phenomena that may be dominant when dealing with uncertainties. We also see that within this point of view, a multiscale description defines implicitly a theory of data measurement: what do we measure exactly in the laboratory when we perform a single phase permeability measurement or a two phase displacement: a local property or an averaged one? This question is essential for well tests interpretation and most reservoir scale measurements. In particular, how do data correlations such as Willi or Gassmann's, (Boyer and Mari, 1994) that are calibrated in the laboratory scale survive to upscaling? This major conceptual and practical issue was first recognized by Cushman (1985) and is very close in spirit to modern renormalisation group point of view (Goldenfeld, 1992). We will see that this kind of consideration has deep consequences even on the numerical simulations.

Another important debate is stochastic versus deterministic descriptions (Dagan, 1989). A more and more popular approach to take into account the fact that our knowledge of the subsurface is intrinsically incomplete is to view the actual reservoir map as being one realisation of a random process. This means that in addition to the uncertainty of our measurements, we model uncertainties that are related to our doubts about the geological description of the reservoir. In that case, reservoir simulation becomes intrinsically probabilistic: we are not interested by getting a very accurate result about one single reservoir image that is likely to be wrong, but to predict a mean and variance of the production forecasts. A direct consequence is that considering these uncertainties can help to justify upscaling in practice: if the errors due to upscaling are lower that those due to uncertainties; one can safely use upscaled models (Glimm et al., 2001; Gorell and Basset, 2001; Schaaf et al., 2002).

The goal of this series of papers is to provide the reader with up to date approaches of the multiscale representation of heterogeneous reservoirs. The goal of this first paper is more to present a general philosophy rather than detailed results that will be presented in the companion papers. To simplify, we will not discuss the specific case of fractured reservoirs although most of the presented considerations remain valid in this specific case. The sequence of papers is organised as follows.

In this first paper, we will focus on the general context, as well as introducing some notations and basic concepts.

The second paper of Lin Ying Hu will present the most recent trends in geostatistical representation of heterogeneous reservoirs, and its increasingly frequent coupling with fluid flow modelling.

Next, we will consider in Yann Gautier's contribution single phase problems related to the upscaling of the 
permeability occurring during well tests. We will show the relation between the permeability provided from well test interpretation, and the underlying permeability map. An attempt to determine directly geostatistical properties from well tests will be presented.

The next paper of Vincent Artus and Benoît Notinger will focus about the up scaling of transport phenomena, dealing with immiscible two phase flow, and by addressing the coupling between flow and heterogeneities.

The important practical problem of upscaling flow close to wells will be addressed by Didier Yu Ding.

\section{BASIC PROBLEMS, EQUATIONS AND GOALS OF MODERN RESERVOIR ENGINEERING}

In the whole sequence of papers, we will consider flows in a heterogeneous reservoir that is a $2 \mathrm{D}$ or $3 \mathrm{D}$ domain denoted by $\Omega$ of boundary $\partial \Omega$. To describe single-phase flow, we assume that Darcy's law holds (Marle, 1981):

$$
u(\mathbf{r})=-\frac{k(\mathbf{r})}{\mu} \cdot \nabla p(\mathbf{r}, t)
$$

Here, $\mathbf{r}$ denotes the position vector, $\mathbf{k}(\mathbf{r})$ the permeability tensor value at point $\mathbf{r}, \mu$ the fluid viscosity, $\rho(\mathbf{r}, t)$ the local mass density and $f(\mathbf{r}, t)$ a source term (generally Dirac like functions whose support coincide with the position of the wells). The global mass balance equation reads:

$$
\frac{\partial \rho(\mathbf{r}, t)}{\partial t}+\nabla \cdot(\rho(\mathbf{r}, t) u(\mathbf{r}, t))=f(\mathbf{r}, t)
$$

Finally, to get a well posed problem, one must relate the variations of the local density $\rho(\mathbf{r}, t)$ to the pressure $p(\mathbf{r}, t)$ using thermodynamic relations. In particular, after some classical assumptions the standard diffusion equation governing pressure depletion may be derived:

$$
\phi(\mathbf{r}) c_{t} \frac{\partial p(\mathbf{r}, t)}{\partial t}=\nabla \cdot\left(\frac{k(\mathbf{r})}{\mu} \nabla p(\mathbf{r}, t)\right)+Q(\mathbf{r}, t)
$$

Here, $\phi(\mathbf{r})$ denotes the local porosity, and $c_{t}$ the total compressibility (Daviau, 1986).

The transport of a passive tracer of local concentration $c(\mathbf{r}, t)$, is described by means of the classical convection diffusion equation:

$$
\phi \frac{\partial c(\mathbf{r}, t)}{\partial t}+\nabla \cdot(c(\mathbf{r}, t) u(\mathbf{r}, t))=\nabla \cdot(d(u) \cdot \nabla c(\mathbf{r}, t))
$$

Here, $\mathbf{u}(\mathbf{r}, t)$ and $\mathrm{c}(\mathbf{r}, t)$ are uncoupled, $\mathbf{d}(\mathbf{u})$ is the local dispersivity tensor that is supposed to depend on $\mathbf{u}(\mathbf{r}, t)$. This tensor comes itself from an averaging of the small scale (grain size) heterogeneities of the porous medium. The corresponding dispersivity tensor is in practice considerably greater than the tracer molecular diffusion.
In the case of two phase flow, we will consider the simplest case where capillary pressure as well as gravity effects are neglected:

$$
\begin{gathered}
\mathbf{u}_{i}=-\frac{\mathbf{K}(\mathbf{r}) k r_{i}(S)}{\mu_{i}} \cdot \nabla p(\mathbf{r}, t) \\
u(\mathbf{r}, t)=-\mathbf{K}(\mathbf{r}) \lambda(S(\mathbf{r}, t)) \nabla p(\mathbf{r}, t) \\
\nabla \cdot u=-\nabla \cdot(\mathbf{K}(\mathbf{r}) \lambda(S(\mathbf{r}, t)) \nabla p(\mathbf{r}, t))=0 \\
\frac{\partial S(\mathbf{r}, t)}{\partial t}+\nabla \cdot\left[\frac{u(\mathbf{r}, t)}{\phi} \varphi(S(\mathbf{r}, t))\right]=0 \\
\lambda(S)=\frac{k r_{1}(S)}{\mu_{1}}+\frac{k r_{2}(S)}{\mu_{2}}
\end{gathered}
$$

In general, the basic goal of reservoir engineering is to be able to predict the overall production forecasts of the considered reservoir as a function of both available reservoir and fluid "natural" data, whose set is denoted collectively by $\{\mathbf{x}\}$, and exploitation data such as the position and orientation of any kind of wells, a water or gas injection scheme, etc., denoted collectively by $\{\mathbf{y}\}$. The production forecast that consists in general in some oil gas and water rates measured at different times and well locations is denoted collectively by $\mathbf{F}(\{\mathbf{x}\},\{\mathbf{y}\})$. F is thus a vector of high dimensionality. The final goal is to recover the most oil at the lowest technical price with minimum risk. Reservoir engineers must provide oil company managers with these data, as well as the related uncertainties. Thus, development decisions can be taken depending on the strategy of the asset managers.

In more formal terms, one wants to estimate the average forecast $\langle\mathbf{F}(\{\mathbf{x}\},\{\mathbf{y}\})\rangle_{\{\mathrm{x}\}}=\langle\mathbf{F}\rangle_{\{\mathrm{x}\}}(\{\mathbf{y}\})$, where the $\{\mathbf{x}\}$ average $\langle\ldots\rangle_{\{\mathrm{x}\}}$ is taken with respect to the whole set of parameters $\{\mathbf{x}\}$ describing the reservoir. The remaining dependence is over the man-controlled exploitation parameters $\{\mathbf{y}\}$. Estimations of the forecast uncertainties $\left\langle\delta \mathbf{F}^{2}(\{\mathbf{x}\},\{\mathbf{y}\})\right\rangle_{\{\mathbf{x}\}}=\left\langle\mathbf{F}^{2}(\{\mathbf{x}\},\{\mathbf{y}\})\right\rangle_{\{\mathbf{x}\}}-\langle\mathbf{F}\rangle_{\{\mathbf{x}\}}{ }^{2}(\{\mathbf{y}\})$ are also needed. The optimal exploitation scheme $\{\mathbf{y}\}^{*}$ is thus the solution of an optimisation problem: $\{\mathbf{y}\}^{*}=\arg \min$. $\{J\{\mathbf{y}\}\}$, where $J\{\mathbf{y}\}$ is a prescribed oil company dependant function of the mean estimation, $\langle\mathbf{F}\rangle_{\{\mathbf{x}\}}(\{\mathbf{y}\})$, and of its uncertainty $\left\langle\delta \mathbf{F}^{2}(\{\mathbf{x}\},\{\mathbf{y}\})\right\rangle_{\{\mathrm{x}\}}$. The oil company strategy specifies $J\{\mathbf{y}\}$. Different economical scenarii may compared by varying the analytical form of $J\{\mathbf{y}\}$.

So far, to compute the optimum $\{\mathbf{y}\}^{*}$ is a formidable task, because we must face at least the following difficulties:

- The parameterisation $\{\mathbf{x}\}$ is a vector of very high dimensionality $N . N$ of order several millions can currently be attained $e . g$. if $\{\mathbf{x}\}$ represents the whole set of permeability values of a high resolution geological representation of the reservoir. 
- The evaluation of $\mathbf{F}(\{\mathbf{x}\},\{\mathbf{y}\})$ is expensive: it is the task of the reservoir simulator. It displays generally highly nonlinear variations with respect to both set of variables $\{\mathbf{x}\}$ and $\{\mathbf{y}\}$.

- The set of parameters $\{\mathbf{y}\}$ is also huge. Having from 10 to 100 control parameters is realistic, so to map directly the variations of $J\{\mathbf{y}\}$ is itself impossible.

The engineer is thus led to find a compromise between simulation time, accuracy and predictability. Evidently, the first idea is to restrict the parameterisation $\{\mathbf{x}\}$, using any field measured data to add information: this is the purpose of reservoir characterisation studies whose ultimate goal is to restrict $\{\mathbf{x}\}$ to regions where both geological, seismic and well test and even multiphase dynamic data are fulfilled. In the language of reservoir engineers, this corresponds to the History Matching (HM) procedure.

\section{FROM THE GEOLOGICAL MODEL TO THE RESERVOIR MODEL. DETERMINISTIC VERSUS STOCHASTIC}

In the beginning of reservoir modelling, we need a geological description allowing a parameterisation $\{\mathbf{x}\}$ to be set up in a suitable and meaningful form. These choices are mainly provided by the geologist expertise, seismic data, analogous outcrop studies and well data if any. In the early 1970's, most reservoir models were geometrically deterministic: a typical choice was the layer-cake model. Here, the $\{\mathbf{x}\}$ parameters were thus the permeability/porosity of each layer and some geometrical data. Recognising that these models were too rough to represent the reservoir internal heterogeneities, the stochastic approach was followed from the 80' to now (Eschard et al., 1991; Galli et al., 1990; Guérillot et al., 1991). The idea is to recognise that $\{\mathbf{x}\}$ is basically unknown and inaccessible, and to use a probabilistic description: this is the period of great progresses in " 2 points" (the meaning of this locution will be precised later) geostatistics and stochastic modelling (Galli et al., 1991; Deutch and Journel, 1992). A lot of software packages that encapsulates in a rather convivial fashion these geostatistical concepts are available, and most major oil companies use them. A great progress came in the 1990's (Tarantola, 1997) when it was recognised that current practices of history matching could be embedded in a probabilistic framework using the Bayesian approach of conditional probabilities.

In the same time, the increasing progress of computers as well as a deeper understanding of the deposition sedimentation process helped geologists to build more and more complex and realistic small scale reservoir images (Grangeon et al., 1997, 1998; Mallet, 1997). This additional information must be used in the actual stochastic approaches: a possible approach is to use "multiple point" geostatistics that is well suited to model complex image characterised by nontrivial high order correlations. This aspect of the question is the purpose of the paper of Lin Ying Hu.

\section{THE BASIC UPSCALING PROBLEM}

To fix the ideas, in the following sections, we will consider the case of a constant porosity reservoir whose isotropic permeability tensor is supposed to be specified. The reservoir is discretised by means of $N$ Cartesian cubic grid blocks of size $\delta x$. The parameter set $\{\mathbf{x}\}$ will thus be identified to the $n$-uplet values $\left\{k_{1}, k_{2}, \ldots k_{n}\right\}$ of the grid block permeabilities of the reservoir.

The standard way of solving the diffusivity equation is to use a finite volume formulation: for each generic gridblock $i=1, n$, integrate the diffusivity equation, and integrate by parts to get:

$$
\phi c_{t} \delta x D \frac{\partial p_{i}(t)}{\partial t}=\sum_{j \text { neighbour } i} \int_{\partial_{i j}} n \cdot \frac{k(\mathbf{r})}{\mu} \nabla p(\mathbf{r}, t) d r+Q_{i}(t)
$$

Here, $p_{i}(t)$ is the average pressure of block $i$, and $\partial_{i j}$ denotes the boundary between blocks $i$ and $j$. To get a well posed problem, we must firstly relate the mean flux between two neighbouring grid blocks $i$ and $j$ to the mean pressures $p_{i}(t)$ and $p_{j}(t)$. A classical method is to suppose that in fact block $i$ corresponds to a homogenous block whose permeability $k_{i}$ would be uniformly equal to $k_{i}$, and similarly for block $j$ with $k_{j}$. Writing thus the flux continuity, and eliminating the intermediate pressure $p_{i j+1 / 2}$ we get finally the standard scheme that reads:

$$
\begin{gathered}
\phi c_{t} v_{i} \frac{\partial p_{i}(t)}{\partial t}=\sum_{j \text { neighbour } i} t_{i j}\left(p_{j}(t)-p_{i}(t)\right)+Q_{i}(t) \\
t_{i j}=\frac{2 k_{i} k_{j}}{k_{i}+k_{j}} \times \frac{S_{i j}}{l_{i j}}, v_{i}=i \text { th grid block volume }
\end{gathered}
$$

After that, any time discretization scheme can be chosen to solve numerically this set of differential equations, and boundary conditions must be accounted for.

Notice that in practice, the righ hand side, rhs, of the equation contains both information about the grid block averaged source term, and also source terms arising from the Dirichlet or Neuman boundary conditions.

Now, consider the classical up scaling problem, as sketched on Figure 1: one wants to solve the same problem as before, using a coarse grid that is supposed to be also Cartesian with $N$ grid blocks $V_{I}, I=1, N$ of size $\Delta X$.

Now, as a matter of convenience, capital letters will denote up scaled quantities while lower case represent small scale data. If the medium was homogeneous, one would get discrete equations under the form:

$$
\phi c_{t} V_{I} \frac{\partial P_{I}(t)}{\partial t}=\sum_{J \text { neighbour I }} T_{I J}\left(P_{J}(t)-P_{I}(t)\right)+Q_{I}(t)
$$




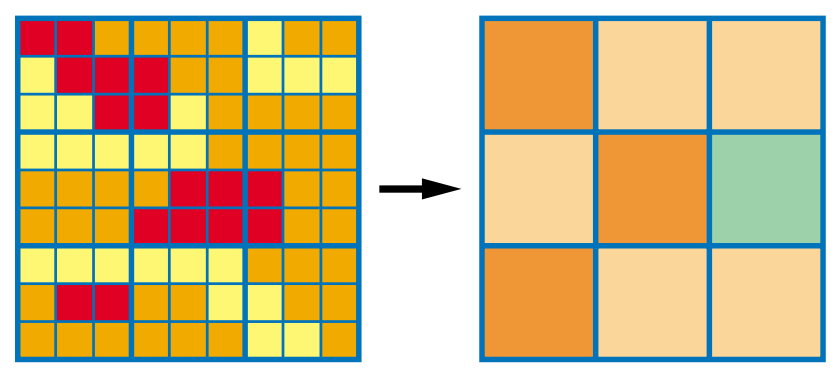

Figure 1

Typical applied upscaling problem. The fine grid of the left must be coarsened to get the grid on the right. Here, each coarse block is made of $3 \times 3$ fine grid blocks. The basic problem is to define the permeability of the coarse block as a function of the fine grid block permeabilities.

In the heterogeneous case, we suppose that we are using a simulator solving equations written under the same form.

Here the $T_{I J}$, given by $T_{I J}=\frac{2 K_{I} K_{J}}{K_{I}+K_{J}} \times \frac{S_{I J}}{l_{I J}}$ will be related to the high resolution permeability map via the values of $K_{I}$ and $K_{J}$, which in turn depend on the small scale permeability map. The quantity $V_{I}$ is its volume. Let us introduce the large scale averaged pressure defined by:

$$
\bar{P}_{I}(t)=\frac{1}{V_{I}} \int_{V_{I}} p(r, t) d r=\frac{1}{N_{I}} \sum_{i \in i(I)} p_{i}
$$

The basic upscaling problem is thus to find a set of $N$ coarse scale permeability tensors $\mathbf{K}_{I}$ such that the $P_{I}(t)$ solution of (10) will be "as close as possible" to the mean coarse block pressure $\bar{P}_{I}(t)$ that could be computed having the fine grid simulation. The norm denoted by $\|\ldots\|$ is for example written in the form:

$$
\left\|\bar{P}_{I}(t)-P_{I}(t)\right\|^{2}=\int d t \sum_{I}\left(\bar{P}_{I}(t)-P_{I}(t)\right)^{2}
$$

This means that we expect that the solution of the coarse scale pressure equations must be as close as possible to the volume averaged pressures. If one is more interested by having a good accuracy on the local rates, $k \nabla p$, this can be accounted for by choosing a more suitable norm involving this quantity. Notice that so far, we have not specified if we were following a stochastic or a deterministic approach. At this stage, this formulation of up scaling does not need this information. Some comments have to be given, because some hidden assumptions have already been retained.

- We have assumed that Darcy's law remains valid at coarse scale. this is the most severe assumption. So far, we only know that we must respect the linearity of the underlying equations. We will discuss this point in more details in Section 5.

- Working at the level of the numerical scheme, what is needed are the coarse scale transmissibilities $T_{I J}$. It could be possible to compute directly these quantities avoiding the intermediate permeability upscaling step. Notice that we can work directly at the level of transmissibilities $T_{I J}$ without relying to any intermediate coarse permeabilities. Such an approach was employed to improve the accuracy of upscaling by Romeu and Nœtinger (1994), or to incorporate near well effects by Ding (1997), or to upscale complex Corner Point type grids having complex shapes by Urgelli (1998). This will be discussed in Section 6, and in Ding paper.

- Even starting from isotropic permeability maps, the resulting permeability should be an anisotropic tensor, because it incorporates information about the geometry.

- Upscaled permeabilities $K_{I}$ should depend on the whole set of small scale values $\left\{k_{1}, k_{2}, \ldots k_{n}\right\}$. In practice, one seeks a formula such that $K_{I}=K_{I}(\{\mathrm{k}\} i \in i(I)\}$, so the dependence of $K_{I}$ is restricted to the small scale values pertaining to the coarse grid block itself. This corresponds to the standard procedure when one considers the coarse block $I$ as being isolated and devise a procedure to define the so called "equivalent permeability tensor" of the block. Some attempts were proposed to overcome this limitation by incorporating immediate neighbouring blocks. No definitive improvement was attained, so we will not pursue this discussion, keeping in mind the traditional approach.

- Finally, the criterion involving the norm $\|.$.$\| cannot be$ fulfilled for every kind of flows. The standard practice implies considering some typical families of flows that occur frequently in oil reservoirs: the linear or the radial case corresponding to the flow toward a well. This will be presented in next section.

The immediate practical interest of this set of assumptions is that exactly the same simulator can be used at both scales. It avoids any specific developments and the user has to concentrate only on the choice of the "upscaling function" $K_{I}\left(\left\{k_{i}\right\} \in i(I)\right\}$.

\section{ABOUT THE CHOICE OF THE UPSCALING FUNCTION}

We are now led to find a "best estimation" of the upscaling function $K_{I}(\{\mathrm{k}\} i \in i(I)\}$ that for simplicity will be denoted with obvious notation $\mathbf{K}(k)$. To discuss this point, several points of view can be adopted. Notice that in practice, we can restrict ourselves to several class of flows: steady state quasi linear and steady state radial flows. Radial flows will be 
considered in the paper by Ding. Here, we will consider the linear flow case by looking at the following problem:

$$
\text { Solve } \nabla \cdot\left(\frac{k(\mathbf{r})}{\mu} \nabla p(\mathbf{r}, t)\right)=\nabla \cdot\left(\frac{k(\mathbf{r})}{\mu}\langle\nabla p\rangle\right)
$$

with $p=0$ at infinity. The vector $\langle\nabla p\rangle$ models a forcing term that corresponds to an imposed overall pressure gradient. After discretisation, on a rectangular domain, we get, for $i=1, n$ :

$$
\sum_{j \text { neighbour } i} t_{i j}\left(p_{j}-p_{i}\right)=\sum_{j \text { neighbour } i} t_{i j} n_{i j} \cdot\langle\nabla p\rangle
$$

where $\mathbf{n}_{i j}$ is the outward normal of $\partial_{i j}$. Boundary conditions (imposed pressure and no flow boundaries, periodic, etc.) must be specified to have a well defined problem having a unique solution. The large scale problem reads:

$$
\sum_{J \text { neighbour I }} T_{I J}\left(P_{J}-P_{I}\right)=\sum_{J \text { neighbour I }} T_{I J} n_{I J} \cdot\langle\nabla p\rangle
$$

The basic goal of upscaling is to provide a simple way of computing the $T_{I J}$ in order that the computed pressures $P_{I}$ and inter coarse grid blocks fluxes $\Phi_{I J}=T_{I J}\left(P_{I}-P_{J}\right)$ are "as close as possible" to the reference ones. To reach this goal, several different approaches may be followed.

\section{Approach A}

The simplest idea is to consider that $K_{I}$ is the value of the permeability evaluated at the coarse grid block center. So, we have $K_{I}=k_{i l}$, where $i_{I}$ is the small scale index labelling the fine grid block that contains the center of the coarse one. This choice is likely to be correct if the small scale permeability is rather smooth at the coarse scale. On the contrary, if it is very heterogeneous, this value may not be representative of the underlying distribution and another approach must be followed. In particular, if the coarse grid block size becomes very much larger than the correlation length, one can observe that the variability of these values should not change at all, because the permeability histogram will not change: the homogenisation effect is completely missed.

\section{Approach B}

A classical approach is to compute the so-called equivalent permeability of each coarse grid block. The idea is to view the coarse grid block as a plug whose permeability in the $D$ space directions, may be measured in the laboratory using standard methods. It yields the permeability of the homogeneous medium that displays the same relation between mean flux and pressure drop. It may be shown that this approach leads to a permeability or even to a permeability tensor that is close in spirit to the solution of the so called "closure problem" that arises when following a more mathematical homogenisation approach, or large scale averaging (Jikov et al., 1995; Quintard and Whitaker, 1994 and references therein) that can be shown to yield equivalent results (Bourgeat et al., 1988). In particular, if the grid size $\Delta X$ is very large when compared to the typical size of the permeability heterogeneity, homogenisation theorems (Jikov et al., 1994) show that the equivalent permeability reaches a limit called the effective permeability tensor $\mathbf{K}_{\text {eff }}$ of the medium. In consequence, we have $K i=\mathbf{K}_{\text {eff }}$. At this scale, the medium behaves as if it was homogeneous, and the effect of the small scale disorder enters only on the value of the mean permeability. The effective permeability tensor $x x$ component $\mathbf{K}_{\text {eff, } x x}$ can be computed solving the following problem that is local in that sense that one must solve boundary value problems only on the considered coarse block:

$$
\begin{aligned}
& \nabla \cdot\left(\frac{k(\mathbf{r})}{\mu} \nabla p(\mathbf{r}, t)\right)=0 \\
& p(\mathbf{r}+\mathbf{L} \hat{\mathbf{x}}, t)=p(\mathbf{r}, t)+\Delta P, r \in \text { inlet } \\
& p(\mathbf{r}+\mathbf{L} \hat{\mathbf{y}}, t)=p(\mathbf{r}, t), r \in \text { faces } / / \text { mean flow } \\
& \quad \mathbf{K}_{\mathrm{eff}, x x}=\frac{1}{L \Delta P} \int_{\text {inlet }} \frac{k(\mathbf{r})}{\mu} \nabla p(\mathbf{r}, t) . n d s
\end{aligned}
$$

$\lambda=\Delta P / L$. The resulting permeability tensor can be shown to fulfil also symmetry and energy requirements. In that case, it is obvious that Approach A would provide completely different results as no any "smoothing" effect can be expected. If the grid size remains small, Approach B would give results close to Approach A.

We will not discuss here some computational details of Approach B: in practice, the boundary conditions can be periodic or linear, etc. If the averaging volume size is sufficiently large, all choices yield the same tensor. A very detailed review may be found in Renard and de Marsily (1997).

\section{Approach C}

This is the opposite of approach A. We consider that $k_{i}$ is a random permeability map. It is thus possible to perform a Monte-Carlo Study (MCS) to compute average pressures, rates etc and even probability densities. The matching criterion becomes that the well rates on the large scale simulation should be equal to the Monte-Carlo average and also their variance, or even the whole density.

In particular, in 2D lognormal case, a well known and very beautiful exact result of Matheron (1967) shows that the equivalent permeability $\mathbf{K}_{\mathrm{eff}}$ is given by the ensemble geometric mean of the permeability: $\mathbf{K}_{\text {eff }}=K_{g}=\exp \langle\ln k\rangle$. This result is obtained mainly without any calculation and uses specific "duality" properties of 2D problems defined by conservation laws.

The practical signification is that $K_{I}$ may be directly replaced by $K_{g}$. This approach is not completely satisfying, because all the underlying variability of the small scale realisation is definitively lost, as now $K_{I}$ and $k_{i}$. are not related at all. In particular, the grid block permeability is no longer random, because it is equal to $K_{g}$. 
An intermediate choice that accounts for the homogenisation effect, and that allows to keep the underlying variability is to replace the ensemble averaged $K_{g}$ by any empirical estimation having the same average, e.g.:

$$
K_{I}=\exp \frac{1}{n_{I}} \sum_{\text {block } I} \ln k_{i}
$$

We have obviously $\left\langle\ln K_{I}>=\ln K_{g}\right.$. The underlying idea is that performing MCS simulations using fine and coarse grids should provide correct estimates of both the mean and variance. Replacing $K_{I}$ by $K_{g}$ provides a correct mean but the variance is missed (it becomes 0). It is in that sense that it is the complete opposite of Approach A which misses completely the homogenisation effect, and transfers the variability independently on the computational grid, which is rather unphysical. Replacing by $K_{I}=\exp 1 / N_{I} \Sigma \ln k_{i}$ is a quite reasonable choice, whose quality can only be tested by extensive (and expansive) MCS, before having a complete stochastic theory. Such Monte-Carlo simulations were performed by Gautier and Nœtinger (1997), who compared the effective permeability obtained using Approach B and the result of Equation (16): an excellent correlation was found in the investigated cases: it is only in the case of non log normal at all permeability distributions that significant difference may be observed. In some sense, Approach $\mathrm{C}$ can be interpreted as being a simplification in the stochastic framework of Approach B.

In most stochastic hydrology literature, this approach is the current practice. One develops stochastic approaches to get an estimator of the average flow, and after, one replaces the resulting answer by empirical estimates to keep the variability. Such an approach was generalised for anisotropic cases (Dagan 1989; Duquerroix et al., 1993), and the problem of facing categorial models remains open.

The main conclusion of this section is that depending on the matching criterion, one can find rather different upscaling functions. Due to the current computing speed, Approach B is actually the more robust and flexible choice that is now recommended, and we can expect to reproduce also the variability of the mean flow. Point $\mathrm{C}$ is important as it

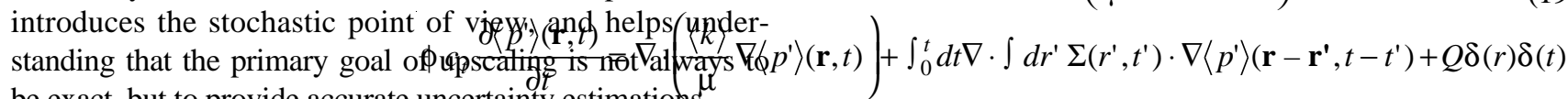
be exact, but to provide accurate uncertainty estimations.

\section{ABOUT PRESSURE TRANSIENTS: THE SELF AVERAGING PROPERTY}

To go further in our investigation of a multiscale description of the flow, let us consider a well test in a 2D heterogeneous reservoir, whose no flow boundaries are supposed to be at infinity. We consider a drawdown well test where the flow rate is equal to $Q$ after $t=0$. The well is supposed to be located at the origin of coordinates. The pressure derivative $\frac{\partial p(r, t)}{\partial t}=p^{\prime}(r, t)$ is shown to obey the following equation (de Marsily, 1981):

$$
\phi c_{t} \frac{\partial p^{\prime}(\mathbf{r}, t)}{\partial t}=\nabla \cdot\left(\frac{k(\mathbf{r})}{\mu} \nabla p^{\prime}(\mathbf{r}, t)\right)+Q \delta(r) \delta(t)
$$

in other words, $p^{\prime}(\mathbf{r}, t)$ is the Green's function of the diffusion problem. In well test, see Daviau (1986), it is common to compute the logarithmic time derivative of the pressure measured at the well that is given by:

$$
t p^{\prime}(r=0, t)=t \frac{d p(t)}{d t}=\frac{\mu Q}{4 \pi k}
$$

The last equality is valid in the homogeneous case, and it is the basis of well test interpretation providing an estimation of the reservoir permeability. In the heterogeneous case, Equation (18) can be used to define an "instantaneous apparent permeability" $k(t)$ that will depend on time (Feitosa, 1993).

The heterogeneous reservoir will be supposed to be a single realisation of a lognormal process of correlation length $l_{c}$.

The permeability map $k(\mathbf{r})=\exp Y(\mathbf{r})$ is supposed to be a stationary lognormal (i.e. it logarithm has a multi-Gaussian distribution) random field having the following properties:

$$
K_{g}=\exp \langle\ln k(r)\rangle, \quad C(r)=\left\langle\ln \frac{k(r)}{K_{g}} \ln \frac{k(r=0)}{K_{g}}\right\rangle
$$

The symbol $\langle\ldots\rangle$ represents the average of the quantity under brackets with respect to all the possible permeability maps. $K_{g}$ is the geometric mean, $C(\mathbf{r})$ is the log permeability correlation function.

Let us consider the quantity $\langle P\rangle(\mathbf{r}, t)$ which is the average of the local pressure that could be obtained by Monte-Carlo simulations. Using perturbation methods arising from statistical physics, it is possible to show that the following equation governs the evolution of the average pressure $\left\langle p^{\prime}\right\rangle(\mathbf{r}, t)$ :

$$
\phi c_{t} \frac{\partial\left\langle p^{\prime}\right\rangle(\mathbf{r}, t)}{\partial t}=\nabla \cdot\left(\frac{\langle k\rangle}{\mu} \nabla\left\langle p^{\prime}\right\rangle(\mathbf{r}, t)\right)
$$

This linear equation has the structure of an integro differential equation, and was derived independently by Indelman (1996), and Notinger and Gautier (1998) who used a Feynman graph approach. The memory kernel $\Sigma(r, t)$ (a second order symmetric tensor) depends explicitly only on the correlation functions of the permeability of arbitrary order. A study of an expansion of $\Sigma(\mathbf{r}, t)$ in a power series of the permeability variance $\sigma$ can be performed using Feynman graphs techniques that provide a systematic method to compute any order term of perturbation theory, and to reorder terms in a physically appealing way (Nœetinger and Gautier, 
1998). Starting from an explicit expression of the average pressure $\langle p(\mathbf{r}, t)\rangle$, these techniques allow to find explicitly the form of the Equation (19) that governs the evolution of the average pressure $\langle p(\mathbf{r}, t)\rangle$. In particular, the kernel $\Sigma(\mathbf{r}, t)$ that is called the "mass operator" in other areas of physics can be computed at any desired order as being the sum of all the corresponding "irreducible graphs". It shows that the average pressure is driven by an equation that has essentially a Darcy structure. It may be shown that up to second order (the "one loop" approximation):

$$
\vec{\Sigma}\left(\mathbf{r}^{\prime}, t^{\prime}\right)=\sigma^{2} \nabla \nabla P_{0}\left(\mathbf{r}^{\prime}, t^{\prime}\right) C\left(r^{\prime}\right)
$$

where $P_{0}\left(\mathbf{r}^{\prime}, t^{\prime}\right)$ denotes the Green's function of the diffusion operator. It shows that this kernel has a typical spatial range equal to $l_{c}$, and a time range of $l_{c}^{2} / D$ which is the typical diffusion time over $l_{c}\left(D=\langle k\rangle / \phi \mu c_{t}\right)$. Equation (19) can be written under a conservation equation form when introducing a local velocity $\mathbf{V}(\mathbf{r}, t)$ as:

$$
\begin{aligned}
& \phi c_{t} \frac{\partial\left\langle p^{\prime}\right\rangle(\mathbf{r}, t)}{\partial t}+\nabla \cdot V(\mathbf{r}, t)=Q \delta(r) \delta(t) \\
V(\mathbf{r}, t)= & -\left\langle\frac{k}{\mu}\right\rangle \nabla\left\langle p^{\prime}\right\rangle(\mathbf{r}, t) \\
& -\int_{0}^{t} d t \int d r^{\prime} \Sigma\left(r^{\prime}, t^{\prime}\right) \cdot \nabla\left\langle p^{\prime}\right\rangle\left(\mathbf{r}-\mathbf{r}^{\prime}, t-t^{\prime}\right)
\end{aligned}
$$

Indelman (1996) showed that this velocity $\mathbf{V}(\mathbf{r}, t)$ is exactly the average of the local Darcy flux defined by $\mathbf{V}(\mathbf{r}, t)=\langle-k / \mu \nabla p(\mathbf{r}, t)\rangle$. This means that the average local rate at point $\mathbf{r}$ is a weighted average of the pressure gradients into the "correlation" zone of $\mathbf{r}$. In the Fourier-Laplace domain, defined by $f(\mathbf{q}, s)=\int_{0}^{\infty} d t \exp (-s t) \int d \mathbf{r} e^{i q \cdot \mathbf{r}} f(\mathbf{r}, t)$, it is possible to generalise the concept of effective permeability by introducing a wave vector dependant effective permeability tensor $\mathbf{K}_{\text {eff, }}(\mathbf{q}, s)$. Using the $(\mathbf{q}, s)$ variables, the complex Relation (22) becomes very simple, taking the form of a simple product involving a wave vector dependant Darcy's law, with $\mathbf{K}_{\text {eff, }}(\mathbf{q}, s)=\langle k\rangle+\mu \Sigma(\mathbf{q}, s)$. The limit of $\mathbf{K}_{\text {eff }},(\mathbf{q}, s)$ for low wave vector, long times $(\mathbf{q}, s) \rightarrow(\mathbf{0}, 0)$ can be identified to $\mathbf{K}_{\text {eff }}$, the steady state effective permeability tensor. This can be justified heuristically, because for long time, we can expect that $\left\langle p^{\prime}\right\rangle(\mathbf{r}, t)$ varies quite smoothly in both $\mathbf{r}$ and $t$ domains, so we can replace it under the integral sign of (19) or (22) by a constant over the correlation volume. So we can write:

$$
V(\mathbf{r}, t) \approx-\left\langle\frac{k}{\mu}\right\rangle \nabla\left\langle p^{\prime}\right\rangle(\mathbf{r}, t)-\left[\int_{0}^{t} d t \int d r^{\prime} \Sigma\left(r^{\prime}, t^{\prime}\right)\right] \cdot \nabla\left\langle p^{\prime}\right\rangle(\mathbf{r}, t)
$$

This result in an effective Darcy's law, with:

$$
\mathbf{K}_{\mathrm{eff}} \approx\langle k\rangle+\mu \int_{0}^{t} d t \int d r^{\prime} \Sigma\left(r^{\prime}, t^{\prime}\right) \underset{t \rightarrow \infty}{\rightarrow}\langle k\rangle+\mu \int_{0}^{\infty} d t \int d r^{\prime} \Sigma\left(r^{\prime}, t^{\prime}\right)
$$

which is well equal to $\mathbf{K}_{\text {eff, }}(\mathbf{q}=\mathbf{0}, s=0)$.
It can also be shown that considering a well test in one realisation, at short time the well test will provide an estimation of the permeability at the well location, at long time the effective permeability $\mathbf{K}_{\text {eff }}$ of the reservoir (Notinger and Gautier, 1998). In between, in can be shown that at first order the apparent permeability is a weighted average of the local permeabilities with a kernel whose support size $R(t) \cong\{2 D t\}^{1 / 2}$, that corresponds to the so-called investigation radius (Blanc et al., 1996) of the well test, is continuously increasing with time (Oliver, 1988; Feitosa et al. 1993; Gautier and Notinger, 1998). At long times, we are thus led naturally to measure the average of a great number of independent events. Central limit theorems can be invoked and we can expect that the apparent permeability will converge "almost surely" to the steady state value (Goldenfeld, 1992; Jikov et al., 1994). It is exactly what happens in practice.

Notinger and Gautier (1998) showed that the apparent permeability $k(t)$ defined by Equation (18), which is a random function depending on time, has the following statistical properties:

$$
\operatorname{Lim}_{t \rightarrow \infty}\langle k(t)\rangle=\mathbf{K}_{\text {eff }},\left\langle(k(t)-\langle k(t)\rangle)^{2}\right\rangle \propto \frac{1}{t}
$$

This means at long time $t$, the apparent permeability is "almost surely" equal to $\mathbf{K}_{\text {eff }}$. In practice, a well test simulation on a sufficiently large single realisation is quite equivalent to perform a Monte-Carlo averaging, at least for large scale phenomena. This is what is called the self averaging property. This result may be qualitatively explained as follows. Considering that the apparent permeability $k(t)$ is a weighed average of the small scale permeabilities over the disk of radius $R(t) \cong\{2 \mathrm{D} t\}^{1 / 2}$ around the well (Oliver, 1988). For large $t$, there are a number $N(t) \approx \pi R(t)^{2} / l_{c}^{2}=2 \mathrm{D} t / l_{c}^{2}$ of independant permeability units in this disk. Central limit theorem shows thus that the variance of $k(t)$ will vary like $1 / N(t) \approx 1 / t$ : this corresponds to the prediction of the detailed calculation of Nœtinger and Gautier (1998). We also understand how the measurement support size may influence the determination of permeability distributions.

Feitosa et al. (1993) and Haas and Nœetinger (1995, 1996), used this way of reasoning to elaborate a method allowing to get reservoir images constrained by observed well test. The idea was to replace the well test simulation by computation of simple averages, yielding a more easy to handle inverse problem.

These results are illustrated in Figure 2, where the results of well test simulations over 5 independent realisations are plotted. We see that after a transient period, all the well tests provide an equivalent permeability. If we were considering an infinite medium, this will be a well defined quantity depending only on the geostatistical structure of permeability, so in the present case its mean and covariance. We can observe some equivalent permeability fluctuations that 


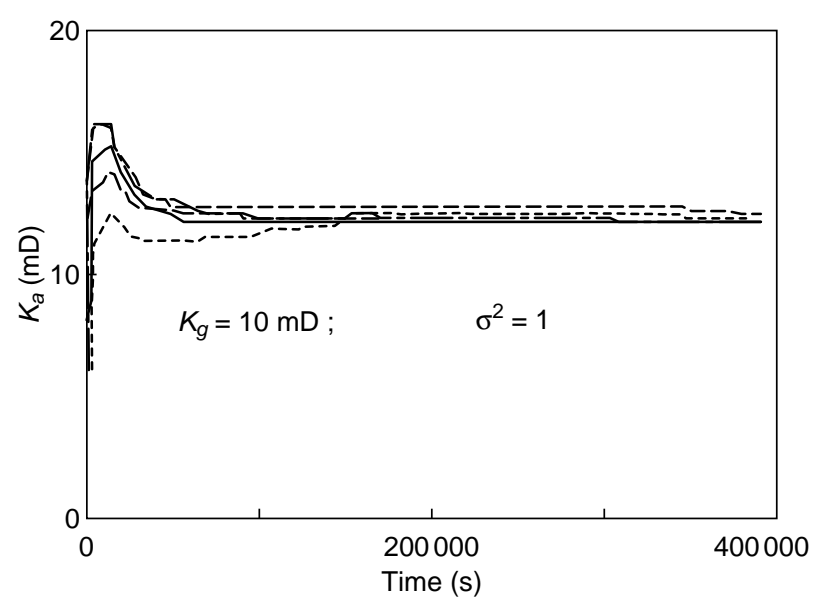

Figure 2

Five "instantaneous apparent permeability" curves corresponding to five well test simulations performed over five independent realisations of a 3D lognormal permeability $K_{g}=10 \mathrm{mD}, \sigma^{2}=1$. The curves are interpreted using Equation (18). The stabilisation of the well test corresponding to the emergence of an homogeneous equivalent medium can be observed after $t=100000 \mathrm{~s}$. The rather low ratio between the size of the reservoir and the correlation length explains the fact that stabilisation of the well test provide realisation dependant equivalent permeabilities.

reflects the finite size of the considered medium. This dependence of well tests on the geostatistical structure of permeability led Gautier and Nœtinger (1998) to try to determine geostatistical parameters such as permeability variance and correlation length using single well test data. These results seemed to be quite difficult to exploit in practice, due to the rather smooth dependence of well test on permeability.

\section{ABOUT NUMERICAL DETERMINATIONS OF THE UPSCALED PARAMETERS}

This issue is quite important because numerical artefacts can provide strongly biased results. Let us come back to the numerical computation of the equivalent permeability of an heterogeneous porous medium. Using a standard approach, and e.g. periodic boundary conditions, we are led to solve a problem having the following structure, to compute the $\mathbf{K}_{\text {eff, } x x}$ component of the effective permeability tensor:

$$
\begin{aligned}
& \nabla .\left(\frac{k(\mathbf{r})}{\mu} \nabla p(\mathbf{r}, t)\right)=0 \\
& p(\mathbf{r}+\mathbf{L} \hat{\mathbf{x}}, t)=p(\mathbf{r}, t)+\Delta P, r \in \text { inlet } \\
& p(\mathbf{r}+\mathbf{L} \hat{\mathbf{y}}, t)=p(\mathbf{r}, t), r \in \text { faces } / / \text { mean flow } \\
& \mathbf{K}_{\text {eff, } x x}=-\frac{1}{L \Delta P} \int_{\text {inlet }} k(\mathbf{r}) \nabla p(\mathbf{r}, t) \cdot n d s
\end{aligned}
$$

Analogous formula can be given for $K_{y y}, K_{x y}$ and $K_{y x}$ components (see Durlovsky, 1991; Romeu, 1994; Gautier and Nœtinger, 1997). The numerical discretisation of these equation leads to the solution of the following linear system:

$$
\begin{aligned}
& \sum_{j \text { neighbour } i} t_{i j}\left(p_{j}-p_{i}\right)=\sum_{j \text { neighbour } i} t_{i j} n_{i j} \cdot \hat{x} \Delta p \\
& \mathbf{K}_{\text {eff }, x x}=\frac{1}{N \Delta P} \sum_{i, j} t_{i j}\left(p_{j}-p_{i}\right)
\end{aligned}
$$

Romeu and Nœtinger (1995) studied numerically and theoretically the preceding formulations on log normal uncorrelated media (i.e. in the present case, situations where the permeabilities of two distinct grid blocks are independent). In all the cases, they observed a systematic difference between the prediction of Matheron (1967), and their numerical results. Lachassagne (1989) was the first to observe this effect, and he called it the systematic bias of numerical simulation. He also observed that a finite element formulation produces an opposite bias: an over estimation of the equivalent permeability. The explanation and quantification of these effects are quite subtle and was the subject of Romeu's $\mathrm{PhD}$ thesis. The basic idea is to recognise that when considering an "uncorrelated" medium, we are quite far from the ideal situation enjoyed by applied mathematicians: the simulation result converges towards the exact solution of the PDE when the grid block size tends to zero. In particular, considering the harmonic weighting rule:

$$
t_{i j}=\frac{2 k_{i} k_{j}}{k_{i}+k_{j}} \times \frac{S_{i j}}{l_{i j}}
$$

low permeability values are systematically advantaged. More generally, Lassachagne (1989) proposed to consider more general weighing formulas written under the form of a power averaging:

$$
t_{i j}^{\omega}=\frac{1}{2}\left(k_{i}^{\omega}+k_{j}^{\omega}\right) \times\left(\frac{S_{i j}}{l_{i j}}\right)^{\omega}
$$

The basic goal is thus to be able to use a "refined" grid in order to fulfil mathematical convergence conditions. The simplest idea was implemented in Romeu and Nœtinger (1995), i.e. dividing each original grid block into $r^{2}$ equal square grid blocks of size $\delta x / r$ having equal permeabilities, as sketched in Figure 3. Notice that this choice is quite arbitrary: we want to model an uncorrelated medium. Following the proposed approach we are implicitly adding information about the short lengthscales (or the high frequencies in the Fourier domain) behaviour of the permeability. This means that we are in fact creating artificial permeability correlations at short lengthscales, so we add information. Another philosophy could be: any refinement scheme such that the equivalent permeability of any 


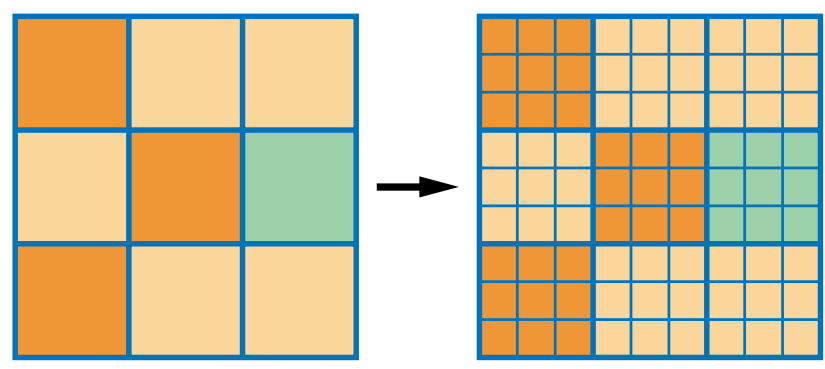

Figure 3

Refining procedure of an "uncorrelated medium". Each original grid block is subdivided into $r \times r$ small grid blocks, whose permeability value is given by the corresponding coarse grid block value.

considered grid block is recovered is correct, it corresponds only to different assumptions about the unknown short distance structure of the medium. In other words, the "uncorrelated medium" is right now mathematically meaningless: we have only a medium whose permeability measurements performed at a given resolution scale did not exhibit any apparent correlation. We will come back on this discussion later.

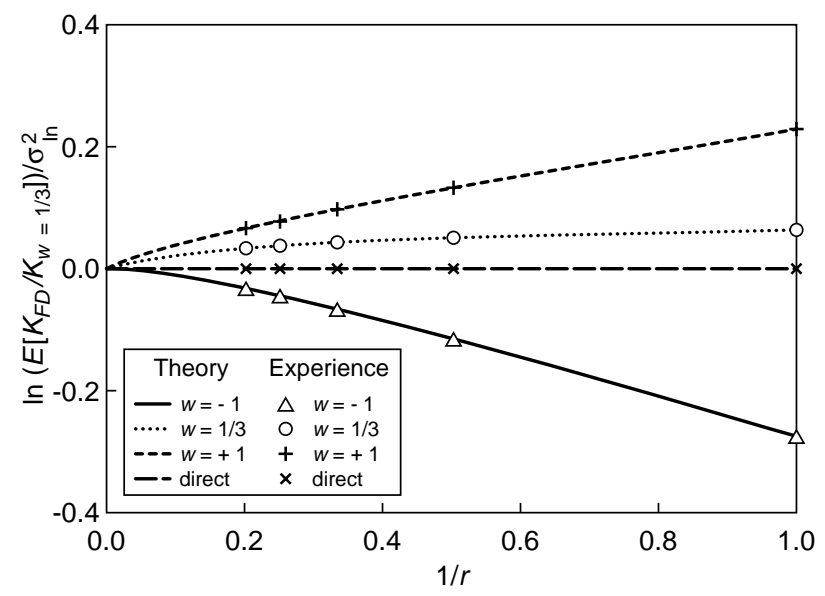

Figure 4

Variations of the large scale permeability normalised by its expected value with the inverse refining index $r$, for a lognormal 3D medium. Each different curve corresponds to different permeability weighing schemes. Here, the variance is small and the agreement between the analytical computation and the numerical experiment is excellent. A 30\% error can be easily attained using a non refined at all calculation $(r=1)$. It can be observed that using harmonically averaged permeabilities and $r=1$ provides that geometric mean for $3 \mathrm{D}$, a wrong result.
In the same paper, we have shown by means of a rather heavy calculation that when $r \rightarrow \infty$, the correct result is recovered. In a next step, we performed a systematic study of these effects using a second order power series expansion of the results of the numerical model as a function of the $\log$ permeability variance. In 3D and 2D, both theoretical and numerical results are summarised in the following curves (Figs. 4, 5 and 6).

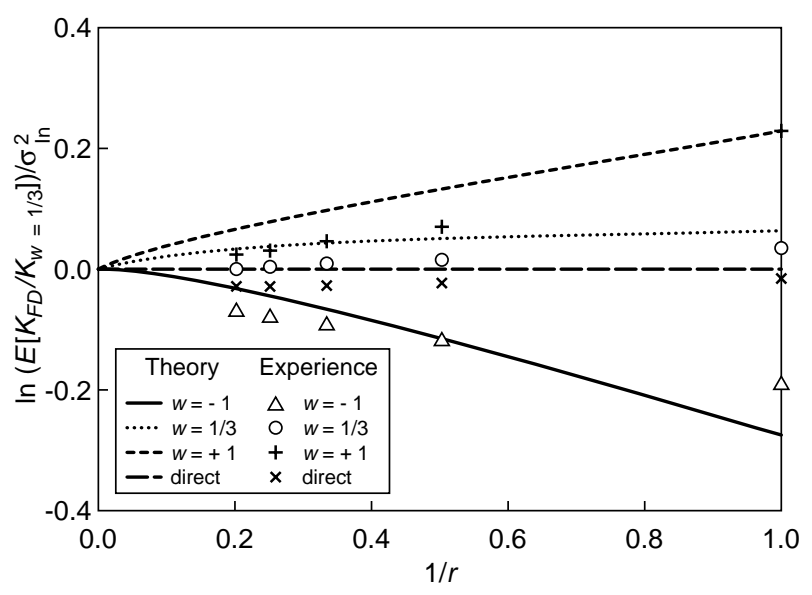

Figure 5

Similar to Figure 4, but this time the log permeability variance is equal to 1. Variations of the large scale permeability normalised by its expected value with the inverse refining index $r$, for a lognormal 3D medium. Each different curve corresponds to different permeability weighing schemes. A $30 \%$ error can be easily attained using a nonrefined at all calculation $(r=1)$.

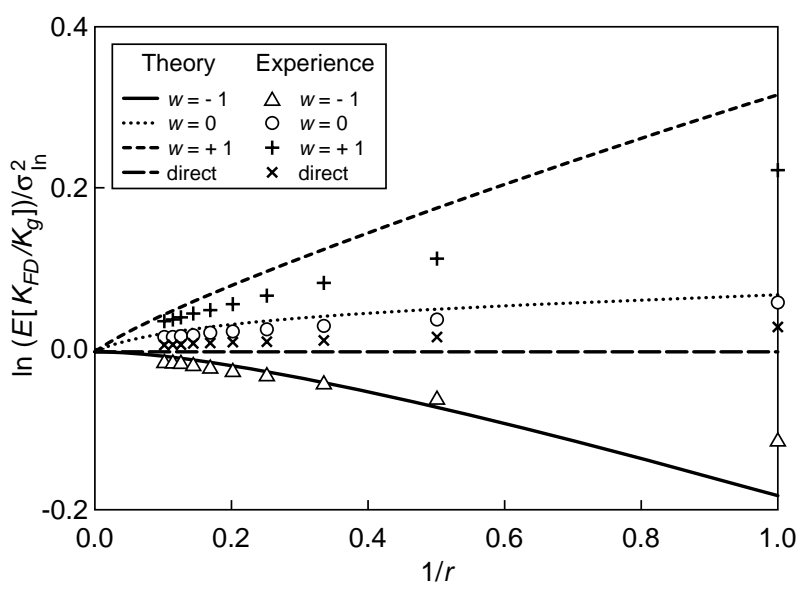

Figure 6

Similar to Figure 5, but this time we consider 2D permeability maps. Variations of the large scale permeability normalised by the geometric mean, its expected value with the inverse refining index $r$. Each different curve corresponds to different permeability weighing schemes. We see that a $30 \%$ error can be easily performed using a non refined at all calculation $(r=1)$. 
The same calculation performed in 3D shows that when $r=1$, the resulting equivalent permeability is close to the geometric average, rather than to the expected $\left\langle k^{1 / 3}\right\rangle^{3}$. This confirms the observation of Warren and Price (1961) that claimed on the basis of numerical simulations that the geometric mean is the correct answer in 3D. Considering correlated random fields, with a correlation length $l_{c}$, shows that it is evidently the discretisation ratio $\delta x / l_{c}$ that must be accounted for.

Finally, we must remember that in order to fulfil accuracy requirements, at least three grid blocks per correlation length are needed to get correct accuracy. If this is not possible, it can be advantageous to use a very coarse grid, where homogenisation effects will play their smoothing role.

\section{ANOTHER POINT OF VIEW: DIRECT UPSCALING OF GEOSTATISTICS, THE FILTERING APPROACH}

\subsection{Background}

We want to come back to our original goal, which is to estimate uncertainties. At this stage, we have replaced the costly Monte-Carlo approach by the solution of a more complex equation whose numerical solution should be simpler. We are close in spirit to our previously presented Approach $\mathrm{C}$ which was shown to miss variability. If our goal is to estimate also uncertainties, we would like to combine both Approaches B and C. In last section, we showed that one must be careful in practice when up scaling grids.

A possible solution is the filtering approach, that is very close in spirit to the LES numerical approach used to model very large Reynolds number fluid dynamics (Germano, 1992; Lesieur and Métais, 1996) or renormalisation group theory in statistical physics (King, 1989; Goldenfeld, 1992; Jaekel and Vereecken, 1997). Consider a stationary lognormal permeability distribution of geometric mean $K_{g 0}$, and of covariance function $C(\mathbf{r})$. For sake of simplicity, we consider an isotropic situation where both permeability tensors and geometric properties are statistically isotropic. A very efficient way of generating realisations $Y(\mathbf{r})=\ln k(\mathbf{r})$ is as follows: consider the Fourier transform of $Y(\mathbf{r})$; denoted by $Y(\mathbf{q})$ defined by $Y(\mathbf{q})=\int d \mathbf{r} e^{-i q \cdot \mathbf{r}} Y(\mathbf{r})$, where $d \mathbf{r}$ denote the natural integration measure and the summation runs over the whole space. One has the Fourier inversion formula $Y(\mathbf{r})=\frac{1}{(2 \pi)^{D}} \int d \mathbf{q} e^{i q \cdot \mathbf{r}} Y(\mathbf{q})$. It can be show that for a stationary multi-Gaussian process $Y(\mathbf{r}), Y(\mathbf{q})$ is also a multiGaussian process which has the following properties:

$\langle Y(\mathbf{q})\rangle=0,\left\langle Y(\mathbf{q}) Y^{\perp}\left(\mathbf{q}^{\prime}\right)\right\rangle=(2 \pi)^{D} C(\mathbf{q}) \delta\left(\mathbf{q}^{\prime}-\mathbf{q}\right)$

Here $\delta\left(\mathbf{q}^{\prime}-\mathbf{q}\right)$ is a $D$ dimensional Dirac delta function, $C(\mathbf{q})$ is the Fourier transform of the covariance function $C(\mathbf{r})$ and $Y$ is the complex conjugate of $Y$. In other words, this means that the Fourier transform diagonalises the covariance matrix. This property remains exact when considering a discretised representation of $Y$ on a regular Cartesian grid. So by taking e.g. $Y(\mathbf{q})=C(\mathbf{q})^{1 / 2} z(\mathbf{q})$ where $z(\mathbf{q})$ is a multiGaussian vector whose covariance matrix is the identity matrix. Coming back to real space by means of an inverse Fourier transform, one can get:

$$
Y(\mathbf{r})=\frac{1}{(2 \pi)^{D}} \int d \mathbf{q} e^{i q \cdot \mathbf{r}} C^{1 / 2}(\mathbf{q}) z(q)
$$

This is the basis of the very efficient FFT MA (Fast Fourier Transform Moving Average) method developed at IFP that combines the advantages of real space and Fourier transform methods accelerated by FFT techniques (Le Ravalec et al., 2000).

\subsection{Filtering Method}

Let us now introduce a cutoff scale $\Gamma\left(\mathrm{m}^{-1}\right)$. We can compute a new random vector denoted $Y_{\Gamma}(\mathbf{q})$ by means of the following definition:

$$
Y_{\Gamma}(\mathbf{r})=\frac{1}{(2 \pi)^{D}} \int_{q<\Gamma} d \mathbf{q} e^{i q \cdot \mathbf{r}} C^{1 / 2}(\mathbf{q}) z(q)
$$

where the integration is restricted to wave vectors whose modulus are smaller than $\Gamma$ (Fig. 7).

So, once $z(\mathbf{q})$ is given, a complete family of $Y_{\Gamma}(\mathbf{q})$ can be obtained depending on the value of the cutoff $\Gamma$. This cutoff plays the role of a filtering parameter: in real space, the proposed operation will correspond to a convolution by a Bessel function whose typical support size would be

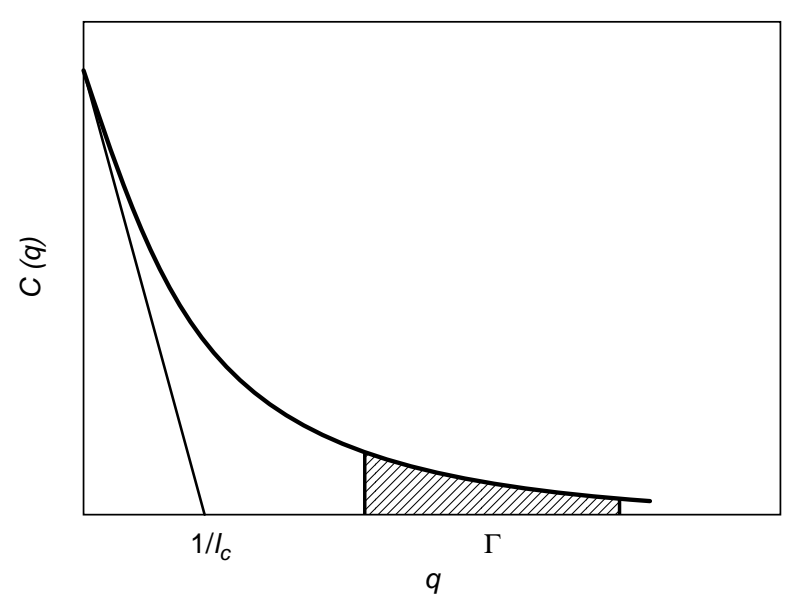

Figure 7

Fourier transform of the covariance function of the $\Gamma$ filtered realisations. Each Fourier mode of wave vector $>\Gamma$ is smoothed out. 

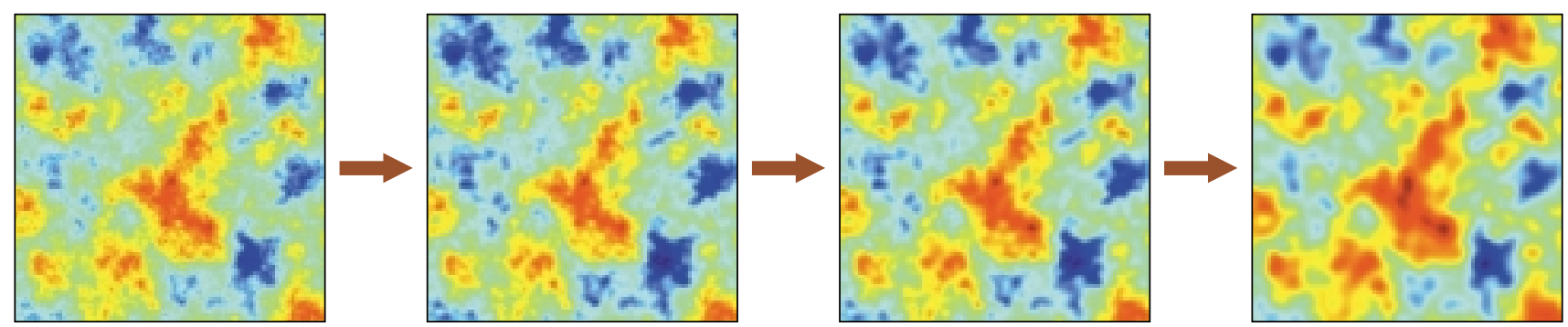

Figure 8

Filtered realisations: due to the basic principle of the FFTMA algorithm, the same set of random numbers is used to generate these different images. These four images differs as Fourier modes of wave vector $>\Gamma$ has been cutoff. The first image is without filtering, and we have $\Gamma_{2}>\Gamma_{3}>\Gamma_{4}$.

$L \cong 2 \pi / \Gamma$. In other words, we are filtering out permeability fluctuations whose frequencies are larger than $\Gamma$. If $\Gamma$ goes to infinity, we recover the original $Y$.

Figure 8 displays the permeability maps corresponding to this process for different values of the cutoff $\Gamma$. The smoothing effect is clear, and corresponds to the intuitive idea of upscaling.

\section{Applications}

The filtering approach could have a broad range of practical applications.

The first application is upscaling and grid coarsening itself. Notice that when high frequencies components are filtered out, the resulting map remains still described by the same number of grid blocks. But, as the filtered realisation is smoother than the original one, it is appealing to coarsen the grid using a typical grid size $\Delta X<1 / \Gamma$. As the permeability map is quite smooth, the upscaling process can be simplified using e.g. the value at the coarse grid block center. This can be observed in Figure 9 where we have added an hypothetical coarse grid, refined close to a well.

In particular, if one wants to set a permeability attribute to an irregular grid, one could attribute to a grid block of characteristic size $1 / \Gamma$, the permeability provided by $k_{\Gamma}(\mathbf{r})$ evaluated at the grid block center.

Once the small scale $z(\mathbf{r})$ map of Gaussian deviates is generated, the evaluation of $k_{\Gamma}(\mathbf{r})$ can be obtained for any $\Gamma$.

Now let us come back to the permeability map by introducing $k_{\Gamma}(\mathbf{r})=K_{g \Gamma} \exp Y_{\Gamma}(\mathbf{r})$. Notice that we introduce a geometric mean $K_{g \Gamma}(\mathbf{r})$ depending on the cutoff parameter $\Gamma$. How can we fix $K_{g \Gamma}$ ?

A simple idea is as follows, Figure 10: consider that we want to compute the equivalent permeability of a large realisation of $Y$ (so $\Gamma=\infty)$ using for example a numerical

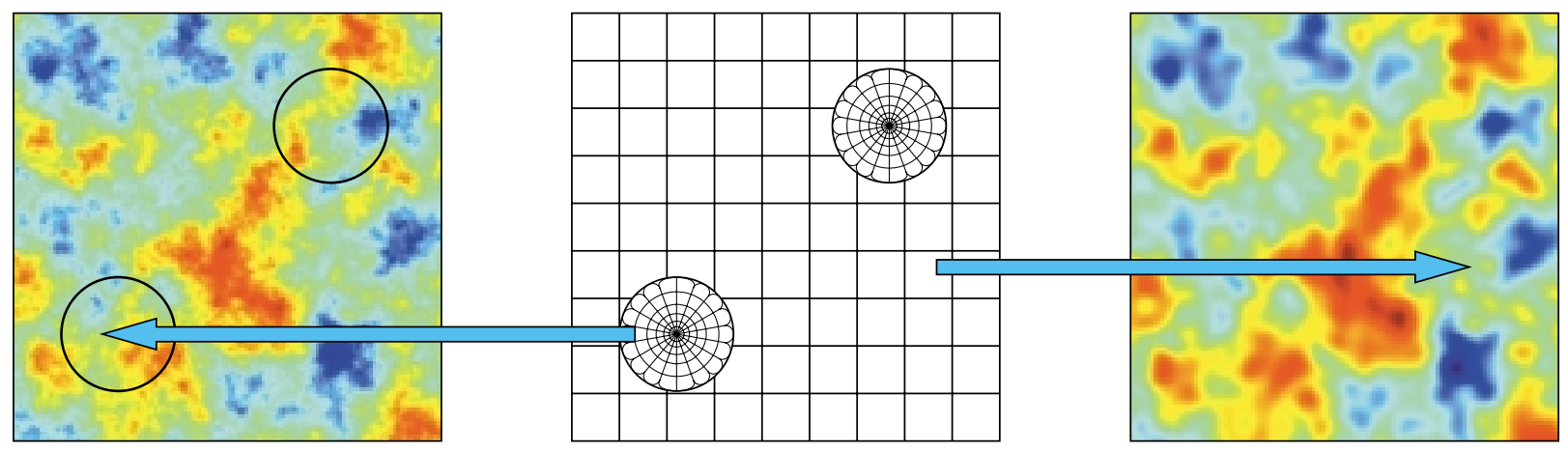

Figure 9

Using the filtering approach to use simultaneously fine and coarse grid data. The upscaling and downscaling aspects are managed simultaneously. 


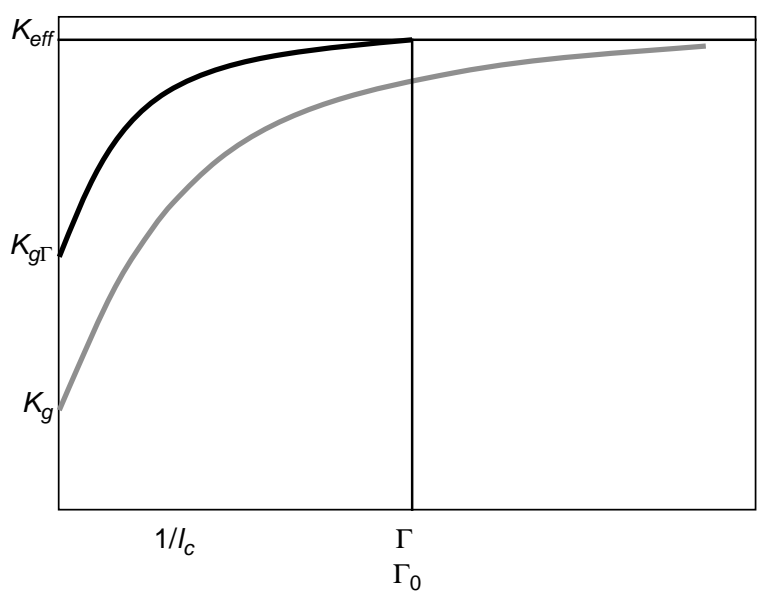

Figure 10

Equivalence criterion between filtered and nonfiltered realisations. The retained criterion is that when computing the average large scale effective permeability $\mathbf{K}_{\text {eff }}$, one should recover equal values. A possible solution is renormalising the mean permeability $K_{g}, K_{g \Gamma}$.

simulation, we obtain thus $\mathbf{K}_{\text {eff }}$, that we will call $\mathbf{K}_{\text {eff }}(\Gamma=\infty)$ $=\mathbf{K}_{\text {eff } \infty}$. Consider now a corresponding filtered realisation with cutoff $\Gamma$ : let us compute now the equivalent permeability of the filtered realisation. It will be proportional to $K_{g \Gamma}$, so we can adjust the geometric mean in order to get hydraulically equivalent realisations providing the same large scale effective permeability. In particular, if we consider the limit of $\Gamma$ tending to zero, as most of the heterogeneities are smoothed out, we will obtain $K_{g \Gamma==>0}=\mathbf{K}_{\text {eff } \infty}$. In other words, the geometric mean of these completely filtered realisations will be equal to the effective permeability of the medium, and as we have smoothed out most of the permeability fluctuations, the variance will be equal to zero. In other words, we will recover the equivalent homogeneous medium.

To summarise, using $k_{\Gamma}(\mathbf{r})$ is equivalent to considering a lognormal random map such that $\left\langle\ln k_{\Gamma}(\mathbf{r})\right\rangle=\ln K_{g \Gamma}$ :

$$
C_{\Gamma}(r)=\left\langle\ln \frac{k_{\Gamma}(r)}{K_{g \Gamma}} \ln \frac{k_{\Gamma}(r=0)}{K_{g \Gamma}}\right\rangle=\frac{1}{(2 \pi)^{D}} \int_{q<\Gamma} d \mathbf{q} e^{i q \cdot \mathbf{r}} C(\mathbf{q})
$$

This provides a useful alternative interpretation of our filtering approach: it can be viewed as a direct upscaling of geostatistics itself.

Depending on the considered support scale, we compute directly the associated geostatistical properties. It can be remarked that when filtering most frequencies $\left(\Gamma \ll 1 / l_{c}\right)$, we have by a direct evaluation of (31):

$$
\sigma^{2} \ln \left(k_{\Gamma}\right) \sim \frac{V^{D}}{(2 \pi)^{D}} \boldsymbol{C}(\mathbf{q}=\mathbf{0}) \Gamma^{D}
$$

where $V^{D}$ is the volume of the sphere of unit radius in $D$ dimensions. This result is another manifestation of the already discussed self-averaging property. To illustrate what happens, let us consider the case of a Gaussian covariance function $C(\mathbf{r})=C(\mathbf{r}=0) \exp -\left(r / l_{c}\right)^{2}$. After an explicit calculation, one gets $\sigma^{2} \ln \left(k_{\Gamma}\right) \sim C(r=0)\left(\Gamma l_{c}\right)^{D}=C(r=0) / n$. So, when $\Gamma$ goes to zero, the variance tends to zero. This expression has a simple interpretation: the quantity $n=\left(\Gamma l_{c}\right)^{-D}$ corresponds to the number of "statistical units", i.e. the number of really independent degrees of freedom that are present in a volume of typical size $L=1 / \Gamma$. It is very appealing from the theoretical point of view, as this quantity is intrinsic, involving only physical quantities. In particular, it does not depend at all on the chosen discretisation. This result is closely related to the central limit theorem and it highlights the homogenisation or smoothing effect.

Concerning the renormalised average permeability, $K_{g \Gamma}$ in 2D, Matheron's theorem shows that $K_{g \Gamma}$ is constant and remains equal to the input geometric mean $K_{g}$ of the distribution.

We can go farther, following an approach that was proposed by Jaekel and Vereecken (1997), to model scale effects on the upscaling of dispersion coefficients. Using again perturbation theory, and a mean-field approximation, it is possible to derive the following differential equation (Notinger, 2000):

$$
\frac{d \log \left(K_{g \Gamma}\right)}{d \Gamma}=\frac{1}{(2 \pi)^{D}}\left(\frac{1}{2}-\frac{1}{D}\right) S_{D} \Gamma^{D-1} C(\Gamma)
$$

with $S_{D}$ equal to the surface of the unit sphere in $D$ dimensions. The mean field approximation consists in the process of considering that fluctuations of permeability of wave vector between $\Gamma$ and $\Gamma+d \Gamma$ interact via an effective homogeneous medium of permeability equal to $K_{g \Gamma}$, rather than an homogenous medium at $K_{g}$. Integrating this ordinary differential equation yields:

$$
\log \left(K_{g \Gamma}\right)=\log K_{g}+\int_{d \Gamma}^{\infty} \frac{1}{(2 \pi)^{D}}\left(\frac{1}{2}-\frac{1}{D}\right) S_{D} \Gamma^{D-1} C(\Gamma)
$$

In particular, when $\Gamma=0$ (i.e. all permeability fluctuations are averaged out), one gets:

$$
\begin{aligned}
& \log \left(K_{g \Gamma=0}\right)=\log K_{g}+\int_{0}^{\infty} d \Gamma \frac{1}{(2 \pi)^{D}}\left(\frac{1}{2}-\frac{1}{D}\right) S_{D} \Gamma^{D-1} C(\Gamma) \\
& \text { so } K_{g \Gamma=0}=K_{g} \exp \left(\frac{1}{2}-\frac{1}{D}\right) C(r=0)
\end{aligned}
$$

We recognize here the Landau-Lifschitz (1960) Matheron's LLM conjecture, which appears as a mean field approximation. It may be written under the equivalent more suggestive form:

$$
\mathbf{K}_{\mathrm{eff}}=\left\langle k^{(1-2 / D)}\right\rangle 1 /(1-2 / D)=\left\langle k^{\omega}\right\rangle 1 / \omega
$$


with $\omega=-1$ in $1 \mathrm{D}$ (harmonic mean), $\omega=0$ in $2 \mathrm{D}$ (geometric mean), $\omega=1 / 3$ in $3 \mathrm{D}$.

Another very interesting point of view is to interpret this renormalised geostatistics as corresponding to measurements performed on a support whose typical size is $1 / \Gamma$. For example, we start from a high resolution permeability map and we consider the observed statistical properties of the permeability of plugs of size $1 / \Gamma>>\delta$. Let us study the geostatistical properties of these data measured on these plugs. Due to homogenisation effect; we expect to observe a reduced variance. It is likely to be close to the proposed ones, because first and second moments are exact. Further tests are needed to check the evolution of geostatistical distributions with the measurement scale. Preliminary tests show that a lognormal medium remains lognormal (a not at all trivial result).

It should be stressed that this point of view is very close to the renormalisation-group approach of quantum field theories (Goldenfeld, 1992), were it is explicitly recognised that it is more important to quantify the evolutions with the measurement scale of the apparent parameters that describes elementary particle (like electrons) and fields (like the electromagnetic field) fundamental interactions rather than the infinite resolution value that is completely inaccessible. This remark can help us to give a precise meaning to the notion of an "uncorrelated" medium i.e. a medium whose correlation length tends to zero discussed in the preceding section. A simple dimensional reasoning shows that the large scale effective permeability $\mathbf{K}_{\text {eff }}$ should not depend on $l_{c}$, because the dimensionless ratio $\mathbf{K}_{\text {eff }} / K_{g}$ cannot depend on a single lengthscale (this can be verified by changing the variable in the steady state diffusion equation). We need another reference lengthscale, that can only be the measurement scale $l_{\text {mes }}$. Considering that $l_{c}$ is smaller than $l_{\text {mes }}$ means only that the measured permeability is uncorrelated at $l_{\text {mes }}$

In the preceding section, we have shown that this point presented some difficulties even in simulations, when the issue is to "refine" the grid representing an uncorrelated medium. The only way to do this correctly is to refine the grid blocks by attributing for example the same permeability to the refined grid blocks. This choice is arbitrary, and probably any refinement procedure such that the overall equivalent permeability of the considered grid block is preserved should be equally acceptable (Romeu and Nœtinger, 1994). So the quantity that is fixed is the apparent $\ln (k)$ variance $\sigma^{2} \ln \left(k_{\Gamma}\right) \sim C(r=0)\left(\Gamma l_{c}\right)^{D}$ with $\left(\Gamma=1 / l_{\text {mes }}\right)$. This means that letting $l_{c}$ tend to zero is correct if the observed variance at the reference scale is kept fixed. The only solution is to assume that the product $C(r=0)\left(l_{c}\right)^{D}$ must remain constant, so $C(r=0)$ varies as $1 / l_{c}^{D}$. This result means simply that due to homogenisation effects, an uncorrelated medium should exhibit heterogeneous behaviour at a finite scale only if the variance is infinite. We recognise here a description of the so called "nugget" effect of geostatisticians (Dagan, 1989). In theoretical physics, this strategy corresponds to the renormalisation procedure of the coupling constants describing the partition function of liquids at the critical point. The basic idea is that completely local values are not measurable in practice (Cushman, 1984). Our measurements are in essence an upscaling process, so what is important is to recover the observed parameters, by adjusting the local values in order to match the observed ones. It is probably in that sense that the famous LLM conjecture becomes exact.

This conjecture has a quite long story. Its simplicity and elegance motivated a great deal of theoretical works. On the more practical point of view, Neumann and Orr (1993) obtained a very good agreement with numerical results even for relatively large log permeability variance (up to 7), and Notinger and Jacquin (1991) tested the formula with real data and they got also an excellent agreement.

In the theoretical point of view, it can easily be shown that this conjecture is exact for $D=1$, for $2 \mathrm{D}$ it was proven by Matheron (1967). In the general case, it is exact up to second order in a series expansion in powers of $C(r=0)$ (see Neumann and Orr (1993) and references therein). Ababou (1994) showed that it was exact for $D=1$ at all order using perturbation theory. Using the general perturbation series, Notinger (1994) showed that a partial summation of a whole perturbation subseries provides LLM result, and he claimed that the formula could be valid for a "vanishing correlation length" case, without giving a precise meaning to this locution.

On the other hand, de Witt (1995) and later independently Indelman and Abramovich (1996), have shown by explicit calculations that are both mathematical tours de force that LLM conjecture is correct up to the next term involving $C(r=0)^{2}$, but that it is incorrect at next order. More precisely, both authors computed explicitly the third order term in $C(r=0)^{3}$ involving interactions between 6 permeability fluctuations. They also show that this term depends explicitly on the whole shape of the covariance function $C(\mathbf{r})$, and not only on the local value $C(\mathbf{r}=0)$. At first sight, this fact has a more severe consequence: it implies that a single purely local averaging formula like LLM cannot exist at all, because non local correlation effects must be accounted for Stepanayants and Teodorovitch (2003) gave similar conclusions.

A important conceptual progress could be attained if it was possible to reconcile these results by giving an hydrodynamic sense to the so called "nugget effect" of geostatisticians. There could be a relation between LLM conjecture, and the definition of the so called vanishing correlation length medium discussed in Section 6. Our filtering approach is a first step towards this goal. A rather analogeous philosophy was followed by Koslov (1993) using homogenisation theory. He found a result similar to LLM for multiscaled permeability maps. This is an indication that 
LLM formula has some importance, and reflects some hidden properties. We think that LLM could be justified asymptotically using the preceding procedure to let $l_{c}$ tending to zero.

We are currently generalising this approach in two directions: anisotropic cases, when the local permeability is an anisotropic tensor, or in the case of geometrical anisotropies. In that case, $k_{\Gamma}(\mathbf{r})$ will become a full tensor $\mathbf{k}_{\Gamma}(\mathbf{r})$. A first result was to obtain an expression of the averaging exponent $\omega$ as a function of the global anisotropy ratio $\alpha=\left(k_{v} / k_{h}\right)^{1 / 2} l_{h} / l_{v}, \omega(\alpha)$. Using second order perturbation theory, we were able to get an analytical determination of $\omega(\alpha)$, in very close agreement with results of numerical simulations (Duquerroix et al., 1991) (Fig. 11). This result is close to estimations provided by other authors using analogous approaches (see e.g. Dagan, 1989):

$$
\omega(\alpha)=\frac{\operatorname{arctg} \alpha}{\pi-\operatorname{arctg} \alpha}
$$

Transposing the filtering approach to anisotropic media leads to solving a set of integro differential equations involving the whole permeability tensor having the a form close to (32). This work is still in progress.

The other important case is to transpose this approach to categorial models. Suppose that we have two different rock types characterised by two permeabilities $k_{1}$ and $k_{2}$. Geostatistical methods provide techniques allowing such maps to be generated. These maps are not lognormal at all, but is it possible to up scale directly geostatistics? Consider now the equivalent permeability probability distribution at a prescribed scale $L=1 / \Gamma$. Such data could be obtained by means of numerical simulations. If $L$ is very small when

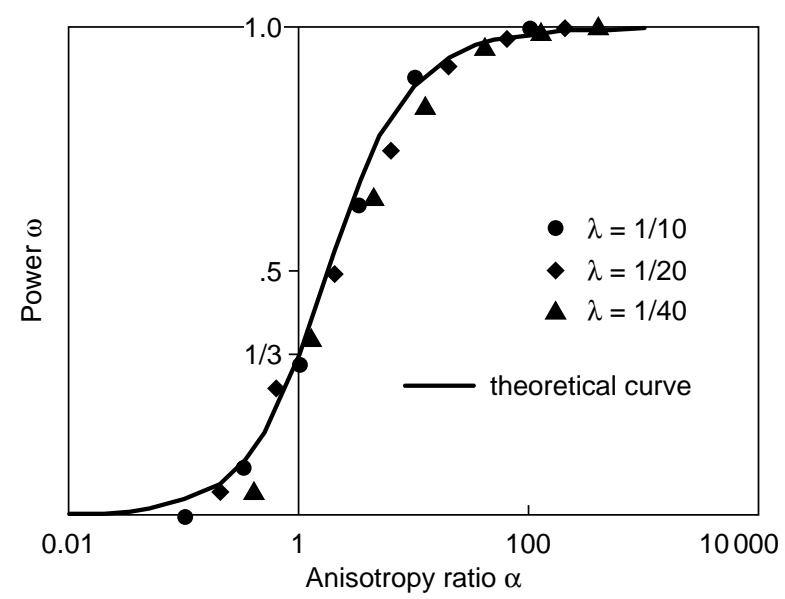

Figure 11

Variations of the averaging exponent $\omega(\alpha)$ as a function of the global anisotropy ratio $\alpha$. A good agreement is obtained between different set of curves and the theoretical prediction. compared to $l_{c}$, we recover the original bimodal distribution. When $L \sim l_{c}$, we can expect to get intermediate values bounded by the two permeabilities, depending on the heterogeneity pattern of the considered medium. For $L$ very large, once again an homogenisation process should occur, and the equivalent permeability probability density distribution should become continuous with a mean tending to $\mathbf{K}_{\text {eff }}$, and a vanishing variance. Such a study could be interesting, mainly to know if there exists a limiting (lognormal?) distribution, and at which scale the discrete character of the original distribution is lost. This would provide some more quantitative insigths about the characteristic size of the so-called "representative elementary volume" whose knowledge is important to predict fluctuations. In addition, the interesting case of a large contrast $k_{1} / k_{2} \rightarrow 0$ would display interesting and non trivial universal critical effects at a percolation threshold depending on the correlation structure of the medium (Stauffer, 1985).

\section{APPLICATIONS TO MULTISCALE HISTORY MATCHING AND MONTE-CARLO STUDIES: TOWARDS AN INTEGRATED DETERMINATION OF UNCERTAINTIES}

Most modern approaches of computer-aided history matching uses the Bayesian paradigm (Tarantola, 1997; Hu et al., 1999; Le Ravalec, 1999). Let us consider that we have observed data denoted collectively by the vector $\mathbf{d}_{\mathrm{obs}}(\mathbf{k})$. The vector $\mathbf{k}$ represents the unknowns (these can be either permeabilities or log permeabilities, porosities, etc.). We suppose that we have a numerical simulator allowing an approximation $\mathbf{d}(\mathbf{k})$ to be computed. To history match a model is equivalent to search for $\mathbf{k}^{*}=\arg \min . J(\mathbf{k})$, the objective function $J(\mathbf{k})$ being given by:

$$
\begin{gathered}
J(k)=\frac{1}{2 \sigma_{d}^{2}}\left(d(k)-d_{o b s}(k)\right)^{2} \\
=\frac{1}{2 \sigma_{d}^{2}}\left(d(k)-d_{o b s}(k)\right)^{2}+\frac{1}{2} \sum_{i, j}\left(k_{i}-k_{p r i o r, i}\right) C_{i j}^{-1}\left(k_{j}-k_{\text {prior }, j}\right)
\end{gathered}
$$

In the Bayesian framework $\sigma_{d}^{2}$ is the variance of measurements + physical and numerical modelling errors, $\sigma_{d}^{2}=\sigma_{\text {meas }}{ }^{2}+\sigma_{\text {mod }}{ }^{2}$. The $C_{i j}{ }^{-1}$ matrix is the inverse of the covariance matrix of $\mathbf{k}$, whose elements are given by $\left.C_{i j}=C\left(\mathbf{r}_{j}-\mathbf{r}_{i}\right)=\left\langle\left(k_{i}-\left\langle k_{i}\right\rangle\right)\left(k_{j}-<k_{j}\right\rangle\right)\right\rangle$ where $\mathbf{r}_{i}$ is the position vector of the center of grid block $i$. In the Gaussian case, and considering that $\mathbf{d}(\mathbf{k})$ is linear, the vector $\mathbf{k}_{\text {prior }}$ can be the a priori average $\langle\mathbf{k}\rangle$, in that case the resulting $\mathbf{k}^{*}$ will be the mean of the posterior distribution, or it can be any $\mathbf{k}_{\text {prior }}$ sampling the prior distribution: in that case $\mathbf{k}^{*}$ will sample the posterior distribution (Oliver et al., 1996).

The practical difficulty is to minimise $J(\mathbf{k})$ because $\mathbf{k}$ is generally of high dimensions, and the model $\mathbf{d}(\mathbf{k})$ represents the reservoir simulator with all the associated complexities. 
In addition, due to nonlinearities of $\mathbf{d}(\mathbf{k}), J(\mathbf{k})$ can display many local minima. Finally, when dealing with multimodal variable, the minimisation process becomes more severe.

Let us see how our filtering approach could help to improve the minimisation of $J(\mathbf{k})$. Let us consider now minimisation of $J$ using filtering. We define a renormalised functional $J_{\Gamma}(\mathbf{k})$ defined by:

$$
\begin{aligned}
J_{\Gamma}(k)=\frac{1}{2 \sigma_{d}^{2}}\left(d(k)-d_{o b s}(k)\right)^{2} \\
+ \\
\quad+\frac{1}{2} \sum_{i, j}\left(k_{i}-k_{p r i o r, i}(\Gamma)\right) C_{\Gamma i j}^{-1}\left(k_{j}-k_{p r i o r, j}\right.
\end{aligned}
$$

Here, $C_{\Gamma i j}=C_{\Gamma}\left(\mathbf{r}_{j}-\mathbf{r}_{i}\right)$ is the covariance matrix of the filtered permeabilities introduced in last section. The idea is to filter out the high frequencies of $\mathbf{k}$, and to minimise $J(\mathbf{k})$ from coarse scales to smaller and smaller scales. Here, $\mathbf{k}_{\text {prior }}(\Gamma)$ represents the renormalised average permeability depending on the filtering parameter $\Gamma$. It can be remarked that working directly with the Fourier components of $\mathbf{k}$, minimising $J_{\Gamma}(\mathbf{k})$ with respect to $\mathbf{k}$ is in fact a minimisation with respect to the low frequency components. This means that we are lowering the dimensionality of the problem in the Fourier domain: it is physically appealing to think that the intrinsic number of unknowns is not the number of grid blocks, but the number of uncorrelated regions that are contained in the reservoir volume, so a ratio $\left(L / l_{c}\right)^{D}$, where $L$ is a typical lengthscale of the reservoir. This number is proportional to the number of wave vectors $q$ for which the correlation matrix $C(\mathbf{q})$ is not equal to zero.

So far, we have not performed any explicit upscaling of the numerical model, we have only simplified the problem parameterisation. The numerical model remains the same, and what is needed is a drastic reduction in the number of minimisation parameters. As we remarked before, since our model is now smoother, grid coarsening becomes easier. We can thus implement it to get the following problem: minimise with respect to $\mathbf{k}, J_{\Gamma}^{\prime}(\mathbf{k})$ defined by:

$$
\begin{aligned}
J_{\Gamma}^{\prime}(k) & =\frac{1}{2 \sigma_{D}^{2}(\Gamma)}\left(d_{u p}(k)-d_{o b s}(k)\right)^{2} \\
& +\frac{1}{2} \sum_{i, j}\left(k_{i}-k_{\text {prior }, i}(\Gamma)\right) C_{\Gamma i j}^{-1}\left(k_{j}-k_{\text {prior }, j}(\Gamma)\right)
\end{aligned}
$$

Here, $\mathbf{d}_{u p}(\mathbf{k})$ represents the simulator working with a coarsened grid, $\sigma_{D}^{2}(\Gamma)=\sigma_{\text {meas }}^{2}+\sigma_{\text {mod }}^{2}(\Gamma)$, where $\sigma_{\text {mod }}^{2}(\Gamma)$ represents now the variance of the numerical model including now upscaling errors. We have now completed the process: we have a restricted parameterisation in the practical real domain, as well as an upscaled reservoir model. Notice that in this presentation, both processes remain quite uncoupled.

Some improvements could be added to this method: e.g. starting with a quite low cutoff $\Gamma$, and once the minimisation is performed, increasing $\Gamma$ in order to add new degrees of freedom representing a more detailed reservoir description and so on, as illustrated on Figure 12.

So far, we have restricted our analysis to Gaussian quantities: how could these ideas be adapted to treat bimodal or multimodal distributions?

This approach may be followed to study uncertainties, by studying carefully the posterior distributions.

Another interesting option that was followed by Schaaf et al. (2002) is to work with $\mathbf{d}_{u p}(\mathbf{k})$ but keeping the fine description for the geological part of the objective function.

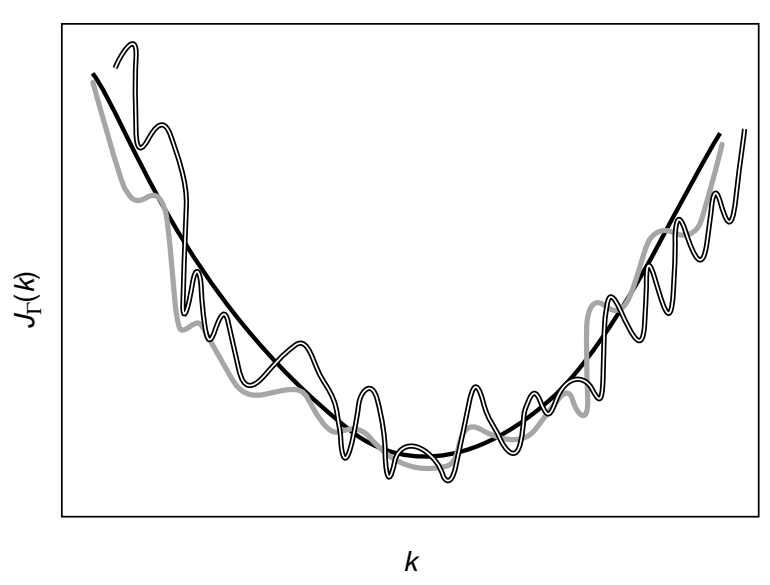

Figure 12

Idea of the multiscale history matching. Each curve display typical variations of the objective function with any degree of freedom. Using strong filtering implies a very smooth curve that can be easily minimised. Details can thus be added if desired by varying the filtering parameter $\Gamma$.

\section{UPSCALING OF TRANSPORT EQUATIONS}

Let us consider now the case of transport equations. We consider the convection-dispersion equation governing the time evolution of the concentration $c(\mathbf{r}, t)$ of a spike of passive tracer (see Marle, 1981 or de Marsily, 1981):

$$
\phi \frac{\partial c(\mathbf{r}, t)}{\partial t}+\nabla \cdot(c(\mathbf{r}, t) u(\mathbf{r}, t))=\nabla \cdot(d(u) \cdot \nabla c(\mathbf{r}, t))
$$

The small scale disorder is modelled through the fluctuations of the local velocity $\mathbf{u}(\mathbf{r}, t)=\langle\mathbf{u}\rangle(\mathbf{r}, t)+\delta \mathbf{u}(\mathbf{r}, t)$. The local dispersion tensor $\mathbf{d}(\mathbf{u})$ is supposed to be known explicitly. The Peclet number comparing the relative importance of transport by convection and dispersion is defined by $\mathrm{Pe}=U l_{c} / d(u)$.

These velocity fluctuations may in turn be due to the underlying permeability disorder. The question is to find the form of the equation governing large scale volume averaged 
concentration $C(\mathbf{r}, t)$ or the ensemble average $\langle c(\mathbf{r}, t)\rangle$. It is possible to derive the following equation, even if the local dispersion tensor $\mathbf{d}(\mathbf{u})$ is equal to zero, corresponding to the purely convective limit at infinite Peclet number:

$$
\phi \frac{\partial\langle c\rangle(\mathbf{r}, t)}{\partial t}+\nabla \cdot(\langle c\rangle(\mathbf{r}, t)\langle u\rangle(\mathbf{r}, t))=\nabla \cdot(D(\langle u\rangle) \cdot \nabla\langle c\rangle(\mathbf{r}, t))
$$

The difficulty is to relate the large scale macrodispersivity tensor $\mathbf{D}(<\mathbf{u}>)$ to the statistical properties of the underlying disorder, and to know at which scale this representation is valid. More detailed studies, using series expansion in a power series of the disorder magnitude, and Feynman graphs reorganisation of the series show that $\mathbf{D}(\langle\mathbf{u}\rangle)$ may be replaced by a memory kernel. A great number of authors, among which major contributions come from Gehlar (1993) and references therein, Dagan (1989) computed $\mathbf{D}(<\mathbf{u}\rangle)$ from the covariance structure of permeability. Both obtained a formula on the form $\left.\mathbf{D}(<\mathbf{u}\rangle) \sim a l_{c} \sigma^{2} u<\hat{\mathbf{u}}\right\rangle\langle\hat{\mathbf{u}}\rangle+b l_{c} \sigma^{2}$ $u(\mathbf{1}-\langle\hat{\mathbf{u}}\rangle\langle\hat{\mathbf{u}}\rangle)$, where $\langle\hat{\mathbf{u}}\rangle\langle\hat{\mathbf{u}}\rangle$ denotes the dyadic tensor build on the average normalised velocity $\langle\hat{\mathbf{u}}\rangle$ and $a$ and $b$ are constant of order unity depending on the precise shape of the covariance function. Using a renormalisation technique close to the one presented in Section 7, Jaekel and Vereecken (1997) proposed a numerical method allowing to account for heterogeneities covering multiple leng scales. This method allows to compute a dispersivity tensor $\mathbf{D}(<\mathbf{u}\rangle$, $L$ ) depending explicitly on the considered averaging scale $L$, and was able to explain quantitatively the observed variations of the apparent dispersivity with the considered lengthscale. More complex heterogeneous reservoirs including multifractal cases were treated by Furtado et al., (1991), Glimm et al. (1992) and Zhang (1992). In that case, anomalous diffusion may arise: the large scale driving equation can have a different form than (39).

Note that when considering miscible flows, where the viscosity of the mixture does vary with concentration, the retroaction between transport equation and the velocity field may induce viscous fingering phenomena. These fascinating phenomena are still the subject of considerable experimental numerical modelling and theoretical efforts. The paper of Vincent Artus will review some of the most important aspects of these efforts in the multiphase case, that remains a major issue for the near future.

\section{CONCLUSIONS AND OPEN PROBLEMS}

In this paper, we have shown that reservoir engineers can gain invaluable information using promising techniques arising from multiple scale approaches, where the entire set of lengthscales characterising the reservoir are treated as a whole. Very practical questions such as history matching the geological model to various data, and uncertainty management can be embedded within a beautiful and useful unifying stochastic framework. Due to the intrinsically complex nature of the real data, change of scale questions are at the heart of this problematic. In particular, the choice of objective functions, and of the optimal parameterisation is closely related to upscaling questions. These upscaling problems may be treated using several different approaches which are mainly equivalent. Numerical methods are now preferred in practice, because in addition to their robustness, they provide directly the link to corresponding down scaling methods, and sensitivity coefficients computations that are needed when dealing with inverse problems provided by history matching questions. Studying the errors associated with upscaling is also an essential issue, as these errors play an explicit role in the objective functions (Eq. 36), to be minimised when solving inverse problems, and also in the uncertainty evaluation.

Many difficult problems are still open concerning upscaling of transport equations, such as tracer or multiphase flow involving highly non linear physical processes. A large effort of classification of equations allowing to devise a "phase diagram" helping the engineer to anticipate the overall flow regime, and the influence of small scale disorder will be an essential step. Of particular interest, are the study of the interaction between heterogeneity and stability considerations. Direct methods using stochastic partial differential equations theory allowing to avoid costly Monte-Carlo simulation would be of considerable interest. Next, specific methods remain to be developed to give practical solutions to these problems, especially for problems involving huge data treatments such as $4 \mathrm{D}$ seismic data interpretation.

\section{ACKNOWLEDGEMENTS}

The authors thanks the permission of IFP to publish these works. Some works were funded by a joint contract with Elf Aquitaine (TOTAL) and Bernard Corre and André Haas are acknowledged. The authors are indebted to many IFP colleagues.

\section{REFERENCES}

Ababou, R. (1994) Solutions of Stochastic Groundwater Flow by Infinite Series, and Convergence of the One-dimensional Expansion. Stochastic. Hydrology and Hydraulics, 8, 139-155.

Ahmadi, A., Labastie, A. and Quintard, M. (1993) Large Scale Properties for Flow Through a Stratified Medium Various Approaches. SPE RE, August, 214-220.

Ahmadi, A. and Quintard, M. (1996) Large Scale Properties for Two Phase Flow in Random Porous Media. Journal of Hydrology, 183, 69-99.

Ahmadi, A. and Nœtinger, B. (1994) A Practical and Operational Method for the Calculation of Effective Dispersion Coefficients in Heterogeneous Porous Media. ECMOR IV, 4th European 
Conference on the Mathematics of Oil Recovery, Röros, Norway, 7-10 June.

Alabert, F.G. (1989) Constraining Description of Randomly Heterogeneous Reservoirs to Pressure Test Data: A Monte-Carlo Study. Paper 19600, SPE 64th Ann. Tech. Conf. and Exh. San Antonio, 207-221.

Arbogast, T. and Bryant, S.L. (2001) Numerical Subgrid Upscaling for Waterflood Simulations. SPE No. 66375, SPE Reservoir Simulation Symposium, Houston, 11-14 February.

Artus, V., Nœtinger, B. and Ricard, L. Dynamics of the Water Oil Front for Two-Phase, Immiscible Flow in Heterogeneous Porous Media. 1 Stratified Media. To be published in Transport in Porous Media.

Artus, V. and Nœtinger, B. (2002) Macrodispersion Approach for Upscaling of Two-Phase, Immiscible Flow in Heterogeneous Porous Media. ECMOR VIII, Freiberg, Germany, 3-6 Sept.

Avellaneda, M. (1996) Homogenization and Renormlization, the Mathematics of Multiple Scale Random Media and Turbulent Diffusion. Lectures in Applied Mathematics, 31, 251-268.

Blanc, G., Guérillot, D., Rahon, D. and Roggero, F. (1996) Building Geostatistical Models Constrained by Dynamic Data-A Posteriori Constraint. SPE 35478, European 3D Reservoir Modeling Conference, Stavanger, Norway, 19-33.

Blanc, G., Nœtinger, B., Piacentino, L. and Helios Reservoir Group (1996) Contribution of the Pressure Moments to the Interpretation of Numerical Simulation of Well Tests. ECMOR V - 5th European Conference on the Mathematics of Oil Recovery, Mining University Leoben, Austria, 3-6 Sept.

Bourgeat, M., Quintard, M. and Whitaker, S. (1988) Éléments de comparaison entre la méthode d'homogéneisation et la prise de moyenne avec fermeture. C.R. Acad. Sci., Paris, 306 2, 463-466.

Boyer, S. and Mari, J.L. (1994) Sismique et diagraphie, Éditions Technip, Paris.

Cardwell, W.T. and Parsons, R.L. (1945) Average Permeabilities of Heterogeneous Oil Sands Trans. American Inst. Min. Pet. Eng., 160, 34-42.

Cherblanc, F. (1999) Étude du transport miscible en milieu poreux hétérogènes: prise en compte du non-équilibre. Thèse, université Bordeaux 1.

Christakos, G., Miller, C.T. and Oliver, D.S. (1993) Stochastic Perturbation Analysis of Groundwater Flow. Spatially Variable Soils, Semi Infinite Domains and Large Fluctuations. Stoch. Hydrol. and Hydrauli., 7, 3, 213-239.

Christakos, G., Hristopulos, D.T. and Miller, C.T. (1995) Stochastic Diagrammatic Analysis of Groundwater Flow in Heterogeneous Porous Media. Water Resources Research, 31, 7, 1687-1703.

Cushman, J.H. (1984) On Unifying the Concepts of Scale, Instrumentation, and Stochastics in the Development of Multiphase Theory. Water Resources Research, 20, 11, 1668-1676.

Cushman, J.H. and Ginn, T.R. (1993) Non Local Dispersion in Porous Media with Continuously Evolving Scale of Heterogeneity. Transport in Porous Media, 13, 1, 123-138.

Dagan, G. (1989) Flow and Transport in Porous Formations Springer Verlag, Berlin.

Daviau, F. (1986) Interprétation des essais de puits, Éditions Technip, Paris.

Deutsch, C.V. and Journel, A.G. (1992) Geostatistical Software Library and User's Guide, Oxford University Press.

Ding, D.Y. and Urgelli, D. (1997) Upscaling of Transmissibility for Field Scale Flow Simulation in Heterogeneous Media. SPE 38016 - Reservoir Simulation, 14th Symposium of the Society of Petroleum Engineers, Dallas, June 8-11, Proceedings, 311-312.
Duquerroix, J.P., Lemouzy, P., Nœtinger, B. and Romeu, R.K. (1993) Influence of the Permeability Anisotropy Ratio on the Large Scale Properties of Heterogeneous Reservoirs. SPE 26648, 68th Annual Tech. Conf. and Exhb. of the SPE, Houston, 29-40.

Durlovsk, L.J. (1991) Numerical Calculation of Equivalent Grid Block Permeability Tensors for Heterogeneous Porous Media. Water Resour. Res., 27, 699-708.

Eschard, R., Doligez, B.D., Rahon, D., Ravenne, C. and Leloch, G. (1991) A New Approach for Reservoirs Description and Simulation Using Geostatistical Methods. Advances in Reservoir Technology, Characterization Modeling and Management.

Feitosa, G.S., Chu, L., Thompson, L.G. and Reynolds, A.C. (1993) Determination of Permeability Distribution From Well Test Pressure Data, SPE 26407.

Furtado, F., Glimm, J., Lindquist, B. and Pereira, F. (1990) Multiple Length Scale Calculus of Mixing Length Growth in Tracer Floods. Kovaritch ed, Proceedings of the Emerging Technologies Conference, Houston TX, Institute for Improve Oil Recovery, 251-259.

Galli, A., Guérillot, D., Ravenne, C. and Heresim-Group (1990) Combining Geology, Geostatistics and Multiphase Fluid Flow for 3D Reservoir Studies. ECMOR II - 2nd European Conference on the Mathematics of Oil Recovery, Proceedings, Arles, Sep. 1114, D. Guérillot, O. Guillon eds., 11-19.

Gautier, Y. and Nœtinger, B. (1997) Preferential Flow-Paths Detection for Heterogeneous Reservoirs Using a New Renormalization Technique. Transport in Porous Media, 26, 1-23.

Gautier, Y. and Nœtinger, B. (1998) Determination of Geostatistical Parameters Using Well-Test Data. SPE 73rd ATCE, 27-30, New Orleans, USA.

Gautier, Y., Blunt, M.J. and Christie, M.A. (1999) Nested Gridding and Streamline-Based Simulation for Fast Reservoir Performance Prediction. Computational Geosciences, 3, 295-320.

Gelhar, W. (1993) Stochastic Subsurface Hydrology, Prentice Hall.

Germano, M. (1992) Turbulence: the Filtering Approach. J. Fluid Mech., 238, 325-336.

Glimm, J., Lindquist, B., Pereira, F. and Peirls, R. (1992) The Multifractal Hypothesis and Anomalous Diffusion. Math. App. Comput., 11, 2, 189-207.

Goldenfeld, N. (1992) Lectures on Phase Transitions and the Renormalization Group, Frontiers in Physics, Addison Wesley.

Gomez-Hernandez, J. (1990) A Stochastic Approach to the Simulation of Block Conductivity Fields Conditioned upon Data Measured at a Smaller Scale, $P h D$, Stanford University.

Gorell, S., and Basset, R. (2001) Trends in Reservoir Simulation: Big Models, Scalable models? Will you please make up your mind? SPE 71 596, ATCE, New Orleans.

Gavalas, G.R. and Shah, P.C. (1976) Reservoir History Matching by Bayesian Estimation. Soc. Pet. Eng. J., 16, 6, 337-350.

Granjeon, D. (1997) Modélisation stratigraphique déterministe Conception et applications d'un modèle diffusif 3D multilithologique. PhD Dissertation, Mémoires Geosciences Rennes, France.

Granjeon, D., Joseph, P. and Doligez, B. (1998). Using a 3D Stratigraphic Model to Optimise Reservoir Description. Hart's Petroleum Engineer International, November, 51-58.

Guérillot, D., Rudkiewicz, J.L., Ravenne, C., Renard, G. and Galli, A. (1989) An Integrated Model for Computer Aided Reservoir, Description: from Outcrop Study to Fluid Flow Simulations. Improved Oil Recovery. 5th European Symposium, Proceedings, Budapest, April 25-27, 651-660.

Guérillot, D.R., Lemouzy, P. and Ravenne, C. (1991) How Reservoir Characterization can help to Improve Production 
Forecasts. Improved Oil Recovery. 6th European Symposium, Proceedings, Stavanger, May 21-23, 3-12.

Guérillot, D.R. and Verdière, S. (1995) Different Pressure Grids for Reservoir Simulation in Heterogeneous Reservoirs. SPE 29148, SPE Symposium on Reservoir Simulation, San Antonio.

Haas, A. and Nœetinger, B. (1996) Stochastic Reservoir Modelling Constrained by Well Test Permeabilities. Fifth International Geostatistics Congress, 22-27 Sept., Wollongong, Australia.

Haas, A. and Nœtinger, B. (1995) 3D Permeability Averaging for Stochastic Reservoir Modelling Constrained by Well Tests, Reservoir Description Forum. The Heriot-Watt and Stanford University, 10-14 Sept., Puebles Hydro, UK.

Horne, R.N. (1990) Modern Well Test Analysis, Petroway.

Hou, T.Y. and Wu, X.H. (1997) A Multiscale Finite Element Method for Elliptic Problems in Composite Materials and Porous Media. J. Comput. Phys., 134, 169-189.

Hou, T.Y., Wu, X.H. and Cai, X. (1999) Convergence of a Multiscale Finite Element Method for Elliptic Problems with Rapidly Oscillating Coefficients. Math. Comput., 227, 913-943.

$\mathrm{Hu}$, L.Y., Blanc, G. and Nœtinger, B. (2001) Gradual Deformation and Iterative Calibration of Sequential Stochastic Simulations. Math. Geol., 33, 475-489.

$\mathrm{Hu}$, L.Y., Blanc, G. and Nœtinger, B. (1996) Estimation of Lithofacies Proportions Using Well and Well-Test Data. SPE 36571, Annual Technical Conference and Exhibition, 6-9 October, Denver, USA.

Hu, L.Y., Le Ravalec, M., Roggero, F., Blanc, G., Nœtinger., B. and Haas, A. (1999) An Overview of the Gradual Deformation Approach for Constraining Gaussian-Related Stochastic Models to Dynamic Data. EAGE/SPE International Symposium on Petroleum Geostatistics, 20-23 April, Toulouse.

Hu, L.Y., Le Ravalec, M., Blanc, G. Corre, B., Haas, A., Nœtinger, B. and Roggero, F. (1999) Reducing Uncertainties in Production Forecasts by Constraining Geological Modeling Using Dynamic Data. SPE 56703, 74th Annual Technical Conference and Exhibition, 3-6 Oct., Houston, USA.

$\mathrm{Hu}$, L.Y., Blanc G. and Nœtinger, B. (1998) Estimation of Lithofacies Proportions by Use of Well and Well-Test Data. SPE Reservoir Evaluation and Engineering, February.

Indelman, P. and Abramovich, B. (1994) A Higher Order Approximation to Effective Conductivity in Media of Anisotropic Random Structure. Water Resources Research, 30, 6, 1857-1864.

Indelman, P. and Abramovich, B. (1995) Effective Permittivity of Log Normal Isotropic Random Media. J. Phys A: Math Gen., 28, 693-700.

Indelman, P. (1996) Averaging of Unsteady Flows in Heterogeneous Media of Stationary Conductivity. J. Fluid. Mech., 310, 39-61.

Jaekel, U. and Vereecken, H. (1997) Renormalization Group Analysis of Macrodispersion in a Directed Random Flow. Water Resources Research, 33, 10, 2287-2299.

Jikov, V.V., Kozlov, S.M. and Oleinik, O.A. (1994) Homogenization of Differential Operators, Springer Verlag.

King, P. (1987) The Use of Field Theoretic Methods for the Study of Flow in Heterogeneous Porous Medium. J. Phys. A. Math. Gen., 20, 3935-3947.

King, P. (1989) The Use of Renormalisation for Calculating Effective Permeability. Transport in Porous Media, 4, 37-58.

Koslov, S.M. (1993) Central Limit Theorem for Multiscaled Permeability. Porous Media Meeting, Birkhauser, 19-33.

Kretz, V., Le Ravalec, M. and Roggero, F. (2002) An Integrated Reservoir Characterization Study Matching Production Data and
4D seismic. SPE - Annual Technical Conference and Exhibition of the Society of Petroleum Engineers, San Antonio, 29 Sep.-2 Oct.

Lachassagne, P. (1989) Estimation des perméabilités moyennes dans les milieux poreux fortement non uniformes étudiés sous l'angle stochastique. Application aux essais de débit en aquifère captif. Thèse de doctorat, École des mines de Paris.

Landau, L.D. and Lifschitz, E.M. (1960) Electrodynamics of Continuous Media, Oxford, Pergamon.

Langlo, P. and Espedal (1994) Macrodispersion for Two-Phase, Immiscible Flow in Porous Media. Advances in Water Resources, 17, 217-316.

Lenormand, R. and Thiele, M. (1996) Determining Flow Equations from Stochastic Properties of a Permeability Field: the MHD model. SPE Journal, 179-190.

Lesieur, M. and Métais, O. (1996) New Trends in Large Eddy Simulations of Turbulence. Ann. Rev. of Fluid Mech., 28, 45-82.

Mallet, J.L. (1997) Discrete Modeling for Natural Objects. Journal of Math. Geology, 29, 2, 199-219.

Marle, C.M. (1981) Multiphase Flow in Porous Media, Éditions Technip, Paris.

Marle, C.M., Simandoux, P., Pacsirszky, J. and Gaulier, C. (1967) Étude du déplacement de fluides miscibles en milieu poreux stratifié. Revue de l'Institut Français du Pétrole, 22, 272-294.

Matheron, G. (1970) La théorie des variables régionalisées et ses applications. Les Cahiers du Centre de morphologie mathématique de Fontainebleau, Fascicule 5.

Matheron, G. (1967) Éléments pour une théorie des milieux poreux, Masson, Paris.

de Marsily, G. (1981) Hydrogéologie quantitative, Masson, Paris. Matheron, G. (1967) Composition des perméabilités en milieu poreux hétérogène: méthode de Schwydler et règles de pondération. Revue de l'Institut Français du Pétrole, 22, 3, 443-466.

Neuman, S.P. and Orr, S. (1993) Prediction of Steady State Flow in Nonuniform Geologic Media by Conditional Moments: Exact non Local Formalism, Effective Conductivities and Weak Approximation. Water Resources Research, 29, 2, 341-364.

Nœtinger, B., Artus, V. and Ricard, L. Dynamics of the Water Oil Front for Two-Phase, Immiscible Flow in Heterogeneous Porous Media. 2 Isotropic Media, to be published in Transport in Porous Media.

Nœtinger, B. (2000) Computing the Effective Permeability of Log-Normal Permeability Fields Using Renormalization Methods. C.R. Acad. des sciences, Sciences de la Terre et des planètes, 331, 353-357.

Notinger, B. and Gautier, Y. (1998) Use of the Fourier-Laplace Transformation and of Diagrammatical Methods to Interpret Pumping Tests in Heterogeneous Reservoirs. Advances in Water Resources, 21, 581-590.

Notinger, B. (1994) The Effective Permeability of a Heterogeneous Porous Medium. Transport in Porous Media, 15, 99127.

Nœtinger, B. and Artus, V. (2002) About Macrodispersion in Immiscible Diphasic Flows in Heterogeneous Porous Media: a Lagrangian Point of View. Second Symposium on Computational Modelling of Multiscale Phenomena, Petropolis, Brazil, 5-9 August.

Nœetinger, B. (1993) A Pressure Moment Approach for Helping Pressure-Transient Analysis in Complex Heterogeneous Reservoirs. SPE 26466, 68th Annual Technical Conference and Exhibition of the Society of Petroleum Engineers, 3-6 Oct., Houston, USA.

Nœtinger, B. and Haas, A. (1996) Permeability Averaging for Well-Tests in 3D Stochastic Reservoir Models. SPE 36653, 
Annual Technical Conference and Exhibition, 6-9 October, Denver, USA.

Nœetinger, B. and Jacquin, C. (1991) Experimental Tests of a Simple Permeability Composition Formula. SPE 22841, 66th Annual Technical Conference and Exhibition of the Society of Petroleum Engineers, 6-9 Oct., Dallas, USA.

Oliver, D.S. (1989) The Averaging Process in Permeability Estimation from Well Test Data. SPE 19845, SPE 64th Ann. Tech. Conf. and Exh.

Oliver, D.S., He, N. and Reynolds, A.C. (1996) Conditioning Permeability to Well Test Data. 5th ECMOR Conf. Proc. Leoben, 259-268.

Pianelo, L., Guérillot, D. and Gallouët, T. (2000) Inversion simultanée des données sismiques et des données de production. Oil \& Gas Science and Technology-Revue de l'IFP, 55, 3, 235-248.

Pianelo, L., Guérillot, D., Gallouët T. (2000) Coupled Inversion of Permeability and Acoustical Impedance an Outstanding Data Integration. ECMOR VII-7th European Conference on the Mathematics of Oil Recovery, Baveno, Proceedings, Paper M-16, Sep. 5-8.

Quintard, M. and Whitaker, S. (1994) Transport in Ordered an Disordered Porous Media ii: Generalized Volume Averaging. Transport in Porous Media, 14, 179-206.

Le Ravalec, M., Hu, L.Y. and Nœtinger, B. (2001) Stochastic Reservoir Modeling Constrained to Dynamic Data: Local Calibration and Inference of Structural Parameters. SPE 68883, SPE Journal.

Le Ravalec, M., Hu, L.Y. and Nœtinger, B. (1999) Stochastic Reservoir Model Constrained to Dynamic Data: Local Calibration and Inference of the Structural Parameters. SPE 56556, 74th Annual Technical Conference and Exhibition, 3-6 Oct., Houston, USA.

Le Ravalec, M., Hu, L.Y. and Nœtinger, B. (2000) The FFT Moving Average (FFT-MA) Method: an Efficient Tool for Generating and Conditioning Gaussian Simulations. Math. Geol. 32, 6 .

Romeu, R.K. and Nœtinger, B. (1995) Calculation of Internodal Transmissibilities in Finite-Difference Models of Flow in Heteregeneous Media. Water Resources Research, April, 31, N4, 943-959.

Romeu, R.K., Lara, A.Q., Nœtinger, B. and Renard, G. (1996) Well Testing in Heterogeneous Media: a General Method to Calculate the Permeability Weighting Function. Four Latin
American and Caribbean Petroleum Engineering Conference, 23-26 April, Port-of-Spain, Trinidad and Tobago.

Rubin, Y. and Dagan, G. (1988) Stochastic Analysis of Boundaries Effects on Head Spatial Variability in Heterogeneous Aquifers 1 Constant Head Boundary. Water Resources Research, 24, 10, 1689-1697.

Ramé, M. and Killough, J.E. (1991) A New Approach to the Simulation of Flows in Highly Heterogeneous Porous Media. SPE 21247, SPE Symposium on Reservoir Simulation, Anaheim.

Renard, Ph. and de Marsily, G. (1997) Calculating Equivalent Permeability: a Review. Advances in Water Resources, 20, 5-6, 253-378.

Saffman, P.G. and Taylor, G. (1958) The Penetration of a Fluid into a Porous Medium or Hele Shaw cell Containing a More Viscous Fluid. Proc. Royal Society of London, A245, 312-329.

Schaaf, T., Mezghani, M. and Chavent, G. (2002) Direct Conditioning of Fine-Scale Facies Models to Dynamic Data by Combining the Gradual Deformation and Numerical Upscaling Techniques. Proc. 8th European Conference on the Mathematics of Oil Recovery, Freiberg, Germany.

Stauffer, D. (1985) Introduction to Percolation Theory, Taylor and Francis, London and Philadelphia.

Stepanayants, Y.A. and Teodorovitch, E.V. (2003) Effective Hydraulic Conductivity of a Randomly Heterogeneous Porous Medium. Water Resour. Res., 39, 3, 1065.

Tarantola, A. (1987) Inverse Problem Theory, Elsevier.

Urgelli, D. (1998) Upscaling of Transmissibility Applied to Corner Point Geometry. Paper SPE 52063, SPE - Society of Petroleum Engineers European Petroleum Conference, The Hague, Oct. 20-22.

Verdière, S. and Guérillot, D. (1996) Dual Mesh Method for Multiphase Flows in Heterogeneous Media. 5th European Conference on the Mathematics of Oil Recovery, Leoben, Austria, September 3-6.

Warren, J.E. and Price, H.S. (1961) Flow in Heterogeneous Porous Media. SPE Journal, 1, 153-169.

de Witt, A. (1995) Correlation Structure Dependance of the Effective Permeability of Heterogeneous Porous Media. Physics of Fluids, 7, 11, 2553-2562.

Zhang, Q. (1992) A Multiple Scale Theory of the Anomalous Mixing Length Growth for Tracer Flow in Heterogeneous Porous Media. J. Stat. Phys., 505, 485-501.

Final manuscript received in April 2004 